Algebraic $8 \mathcal{G}$ Geometric $\mathcal{T}_{\text {opology }}$

Volume 4 (2004) 347-398

Published: 8 June 2004

ATG

\title{
Noncommutative knot theory
}

\author{
TiM D. COCHRAN
}

\begin{abstract}
The classical abelian invariants of a knot are the Alexander module, which is the first homology group of the the unique infinite cyclic covering space of $S^{3}-K$, considered as a module over the (commutative) Laurent polynomial ring, and the Blanchfield linking pairing defined on this module. From the perspective of the knot group, $G$, these invariants reflect the structure of $G^{(1)} / G^{(2)}$ as a module over $G / G^{(1)}$ (here $G^{(n)}$ is the $n^{\text {th }}$ term of the derived series of $\mathrm{G}$ ). Hence any phenomenon associated to $G^{(2)}$ is invisible to abelian invariants. This paper begins the systematic study of invariants associated to solvable covering spaces of knot exteriors, in particular the study of what we call the $n^{\text {th }}$ higher-order Alexander module, $G^{(n+1)} / G^{(n+2)}$, considered as a $\mathbb{Z}\left[G / G^{(n+1)}\right]-$ module. We show that these modules share almost all of the properties of the classical Alexander module. They are torsion modules with higher-order Alexander polynomials whose degrees give lower bounds for the knot genus. The modules have presentation matrices derived either from a group presentation or from a Seifert surface. They admit higher-order linking forms exhibiting self-duality. There are applications to estimating knot genus and to detecting fibered, prime and alternating knots. There are also surprising applications to detecting symplectic structures on 4-manifolds. These modules are similar to but different from those considered by the author, Kent Orr and Peter Teichner and are special cases of the modules considered subsequently by Shelly Harvey for arbitrary 3-manifolds.
\end{abstract}

AMS Classification 57M27; 20F14

Keywords Knot, Alexander module, Alexander polynomial, derived series, signature, Arf invariant

\section{Introduction}

The success of algebraic topology in classical knot theory has been largely confined to abelian invariants, that is to say to invariants associated to the unique regular covering space of $S^{3} \backslash K$ with $\mathbb{Z}$ as its group of covering translations. These invariants are the classical Alexander module, which is the first 
homology group of this cover considered as a module over the commutative ring $\mathbb{Z}\left[t, t^{-1}\right]$, and the classical Blanchfield linking pairing. In turn these determine the Alexander polynomial and Alexander ideals as well as various numerical invariants associated to the finite cyclic covering spaces. From the perspective of the knot group, $G=\pi_{1}\left(S^{3} \backslash K\right)$, these invariants reflect the structure of $G^{(1)} / G^{(2)}$ as a module over $G / G^{(1)}$ (here $G^{(0)}=G$ and $G^{(n)}=\left[G^{(n-1)}, G^{(n-1)}\right]$ is the derived series of $G$ ). Hence any phenomenon associated to $G^{(2)}$ is invisible to abelian invariants. This paper attempts to remedy this deficiency by beginning the systematic study of invariants associated to solvable covering spaces of $S^{3} \backslash K$, in particular the study of the higher-order Alexander module, $G^{(n)} / G^{(n+1)}$, considered as a $\mathbb{Z}\left[G / G^{(n)}\right]$-module. Certainly such modules have been considered earlier but the difficulties of working with modules over noncommutative, non-Noetherian, non UFD's seems to have obstructed progress.

Surprisingly, we show that these higher-order Alexander modules share most of the properties of the classical Alexander module. Despite the difficulties of working with modules over non-commutative rings, there are applications to estimating knot genus, detecting fibered, prime and alternating knots as well as to knot concordance. Most of these properties are not restricted to the derived series, but apply to other series. For simplicity this greater generality is discussed only briefly herein.

Similar modules were studied in COT1 [COT2 [CT] where important applications to knot concordance were achieved. The foundational ideas of this paper, as well as the tools necessary to begin it, were already present in [COT1 and for that I am greatly indebted to my co-authors Peter Teichner and Kent Orr. Generalizing our work on knots, Shelly Harvey has studied similar modules for arbitrary 3-manifolds and has found several striking applications: lower bounds for the Thurston norm of a 2-dimensional homology class that are much better than C. McMullen's lower bound using the Alexander norm; and new algebraic obstructions to a 4 -manifold of the form $M^{3} \times S^{1}$ admitting a symplectic structure [Ha].

Some notable earlier successes in the area of non-abelian knot invariants were the Jones polynomial, Casson's invariant and the Kontsevitch integral. More in the spirit of the present approach have been the "metabelian" Casson-Gordon invariants and the twisted Alexander polynomials of X.S. Lin and P. Kirk and C. Livingston $\mathrm{KL}$. Most of these detect noncommutativity by studying representations into known matrix groups over commutative rings. The relationship (if any) between our invariants and these others, is not clear at this time.

Our major results are as follows. For any $n \geq 0$ there are torsion modules

$\mathcal{A}_{n}^{\mathbb{Z}}(K)$ and $\mathcal{A}_{n}(K)$, whose isomorphism types are knot invariants, generalizing 
the classical integral and "rational" Alexander module $(n=0)$ (Sections 2, 3, 4). $\mathcal{A}_{n}(K)$ is a finitely generated module over a non-commutative principal ideal domain $\mathbb{K}_{n}\left[t^{ \pm 1}\right]$ which is a skew Laurent polynomial ring with coefficients in a certain skew field (division ring) $\mathbb{K}_{n}$. There are higher-order Alexander polynomials $\Delta_{n}(t) \in \mathbb{K}_{n}\left[t^{ \pm 1}\right]$ (Section [5). If $K$ does not have (classical) Alexander polynomial 1 then all of its higher modules are non-trivial and $\Delta_{n} \neq 1$. The degrees $\delta_{n}$ of these higher order Alexander polynomials are knot invariants and (using some work of S. Harvey) we show that they give lower bounds for knot genera which are provably sharper than the classical bound $\left(\delta_{0} \leq 2\right.$ genus $\left.(K)\right)$ (see Section 7).

Theorem If $K$ is a non-trivial knot and $n \geq 1$ then $\delta_{0}(K) \leq \delta_{1}(K)+1 \leq$ $\delta_{2}(K)+1 \leq \cdots \leq \delta_{n}(K)+1 \cdots \leq 2 \operatorname{genus}(K)$.

Corollary If $K$ is a knot whose (classical) Alexander polynomial is not 1 and $k$ is a positive integer then there exists a hyperbolic knot $K_{*}$, with the same classical Alexander module as $K$, for which $\delta_{0}\left(K_{*}\right)<\delta_{1}\left(K_{*}\right)<\cdots<\delta_{k}\left(K_{*}\right)$.

There exist presentation matrices for these modules obtained by pushing loops off of a Seifert matrix (Section [6). There also exist presentation matrices obtained from any presentation of the knot group via free differential calculus (Section 13). There are higher order bordism invariants, $\beta_{n}$, generalizing the Arf invariant (Section 10) and higher order signature invariants, $\rho_{n}$, defined using traces on Von Neumann algebras (Section [11). These can be used to detect chirality. Examples are given wherein these are used to distinguish knots which cannot be distinguished even by the $\delta_{n}$. There are also higher order linking forms on $\mathcal{A}_{n}(K)$ whose non-singularity exhibits a self-duality in the $\mathcal{A}_{n}(K)$ (Section [12).

The invariants $\mathcal{A}_{i}^{\mathbb{Z}}, \delta_{i}$ and $\rho_{i}$ have very special behavior on fibered knots and hence give many new realizable algebraic obstructions to a knot's being fibered (Section 9). Moreover using some deep work of P. Kronheimer and T. Mrowka Kr2 the $\delta_{i}$ actually give new algebraic obstructions to the existence of a symplectic structure on 4 -manifolds of the form $S^{1} \times M_{K}$ where $M_{K}$ is the zeroframed surgery on $K$. These obstructions can be non-trivial even when the Seiberg-Witten invariants are inconclusive!

Theorem 9.5 Suppose $K$ is a non-trivial knot. If $K$ is fibered then all the inequalities in the above Theorem are equalities. The same conclusion holds if $S^{1} \times M_{K}$ admits a symplectic structure. 
Section 9 establishes that, given any $n>0$, there exist knots with $\delta_{i}+1=\delta_{0}$ for $i<n$ but $\delta_{n}+1 \neq \delta_{0}$.

The modules studied herein are closely related to the modules studied in COT1. COT2 [CT], but are different. In particular for $n>0$ our $\mathcal{A}_{n}$ and $\delta_{n}$ have no known special behavior under concordance of knots. This is because the $\mathcal{A}_{n}$ reflect only the fundamental group of the knot exterior, whereas the modules of [COT1] reflect the fundamental groups of all possible slice disk exteriors. To further detail the properties of the higher-order modules of COT1 (for example their presentation in terms of a Seifert surface and their special nature for slice knots) will require a separate paper although many of the techniques of this paper will carry over.

\section{Definitions of the higher-order Alexander modules}

The classical Alexander modules of a knot or link or, more generally, of a 3manifold are associated to the first homology of the universal abelian cover of the relevant 3-manifold. We investigate the homology modules of other regular covering spaces canonically associated to the knot (or 3-manifold).

Suppose $M_{\Gamma}$ is a regular covering space of a connected CW-complex $M$ such that the group $\Gamma$ is identified with a subgroup of the group of deck (covering) translations. Then $H_{1}\left(M_{\Gamma}\right)$ as a $\mathbb{Z} \Gamma$-module can be called a higher-order Alexander module. In the important special case that $M_{\Gamma}$ is connected and $\Gamma$ is the full group of covering transformations, this can also be phrased easily in terms of $G=\pi_{1}(M)$ as follows. If $H$ is any normal subgroup of $G$ then the action of $G$ on $H$ by conjugation $\left(h \longrightarrow g^{-1} h g\right.$ ) induces a right $\mathbb{Z}[G / H]$ module structure on $H /[H, H]$. If $H$ is a characteristic subgroup of $G$ then the isomorphism type (in the sense defined below) of this module depends only on the isomorphism type of $G$.

The primary focus of this paper will be the case that $M$ is a classical knot exterior $S^{3} \backslash K$ and on the modules arising from the family of characteristic subgroups known as the derived series of $G$ (defined in Section 1).

Definition 2.1 The $n^{\text {th }}$ (integral) higher-order Alexander module, $\mathcal{A}_{n}^{\mathbb{Z}}(K)$, $n \geq 0$, of a knot $K$ is the first (integral) homology group of the covering space of $S^{3} \backslash K$ corresponding to $G^{(n+1)}$, considered as a right $\mathbb{Z}\left[G / G^{(n+1)}\right]$-module, i.e. $G^{(n+1)} / G^{(n+2)}$ as a right module over $\mathbb{Z}\left[G / G^{(n+1)}\right]$. 
Clearly this coincides with the classical (integral) Alexander module when $n=0$ and otherwise will be called a higher-order Alexander module. It is unlikely that these modules are finitely generated. However S. Harvey has observed that they are the torsion submodules of the finitely presented modules obtained by taking homology relative to the inverse image of a basepoint $\mathrm{Ha}$. The analogues of the classical rational Alexander module will be discussed later in Section 4. These are finitely generated.

Note that the modules for different knots (or modules for a fixed knot with different basepoint for $\pi_{1}$ ) are modules over different (albeit sometimes isomorphic) rings. This subtlety is even an issue for the classical Alexander module. If $M$ is an $R$-module and $M^{\prime}$ is an $R^{\prime}$-module, we say $M$ is (weakly) isomorphic to $M^{\prime}$ if there exists a ring isomorphism $f: R \rightarrow R^{\prime}$ such that $M$ is isomorphic to $M^{\prime}$ as $R$-modules where $M^{\prime}$ is viewed as an $R$-module via $f$. If $R$ and $R^{\prime}$ are group rings (or functorially associated to groups $G, G^{\prime}$ ) then we say $M$ is isomorphic to $M^{\prime}$ if there is a group isomorphism $g: G \longrightarrow G^{\prime}$ inducing a weak isomorphism.

Proposition 2.2 If $K$ and $K^{\prime}$ are equivalent knots then $\mathcal{A}_{n}^{\mathbb{Z}}(K)$ is isomorphic to $\mathcal{A}_{n}^{\mathbb{Z}}\left(K^{\prime}\right)$ for all $n \geq 0$.

Proof of 2.2 If $K$ and $K^{\prime}$ are equivalent then their groups are isomorphic. It follows that their derived modules are isomorphic.

Thus a knot, its mirror-image and its reverse have isomorphic modules. In order to take advantage of the peripheral structure, one needs to use the presence of this extra structure to restrict the class of allowable ring isomorphisms. This may be taken up in a later paper. However in Section [10 and Section [1] respectively we introduce higher-order bordism and signature invariants which do use the orientation of the knot exterior and hence can distinguish some knots from their mirror images.

Example 2.3 If $K$ is a knot whose classical Alexander polynomial is 1, then it is well known that its classical Alexander module $G^{(1)} / G^{(2)}$ is zero. But if $G^{(1)}=G^{(2)}$ then $G^{(n)}=G^{(n+1)}$ for all $n \geq 1$. Thus each of the higher-order Alexander modules $\mathcal{A}_{n}^{\mathbb{Z}}$ is also trivial. Hence these methods do not seem to give new information on Alexander polynomial 1 knots. However, it is shown in Corollary 4.8 that if the classical Alexander polynomial is not 1 , then all the higher-order modules are non-trivial. 
Example 2.4 Suppose $K$ is the right-handed trefoil, $X=S^{3} \backslash K$ and $G=$ $\pi_{1}(X)$. Since $K$ is a fibered knot we may assume that $X$ is the mapping torus of the homeomorphism $f: \Sigma \rightarrow \Sigma$ where $\Sigma$ is a punctured torus and we may assume $f$ fixes $\partial \Sigma$ pointwise. Then $\pi_{1}(\Sigma)=F\langle x, y\rangle$. Let $X_{n}$ denote the covering space of $X$ such that $\pi_{1}\left(X_{n}\right) \cong G^{(n+1)}$ and $\mathcal{A}_{n}^{\mathbb{Z}}(K)=H_{1}\left(X_{n}\right)$ as a $\mathbb{Z}\left[G / G^{(n+1)}\right]$ module. Note that the infinite cyclic cover $X_{0}$ is homeomorphic to $\Sigma \times \mathbb{R}$ so that $\pi_{1}\left(X_{0}\right) \cong G^{(1)} \cong F$. Thus $X_{n}$ is a regular covering space of $X_{0}$ with deck translations $G^{(1)} / G^{(n+1)}=F / F^{(n)}$. Since $\pi_{1}\left(X_{n}\right)=F^{(n)}, H_{1}\left(X_{n}\right)=$ $F^{(n)} / F^{(n+1)}$ as a module over $\mathbb{Z}\left[F / F^{(n)}\right]$. Therefore if one considers $\mathcal{A}_{n}^{\mathbb{Z}}(K)$ as a module over the subring $\mathbb{Z}\left[G^{(1)} / G^{(n+1)}\right]=\mathbb{Z}\left[F / F^{(n)}\right] \subseteq \mathbb{Z}\left[G / G^{(n+1)}\right]$ then it is merely $F^{(n)} / F^{(n+1)}$ as a module over $\mathbb{Z}\left[F / F^{(n)}\right]$ (a module which depends only on $n$ and the rank of the free group). More topologically we observe that $X_{0}$ is homotopy equivalent to the wedge $W$ of 2 circles and $X_{n}$ is (homotopy equivalent to) the result of taking $n$ iterated universal abelian covers of $W$. Let us consider the case $n=1$ in more detail. Here $X_{1}$ is homotopy equivalent to $W_{\infty}$, as shown in Figure 1]

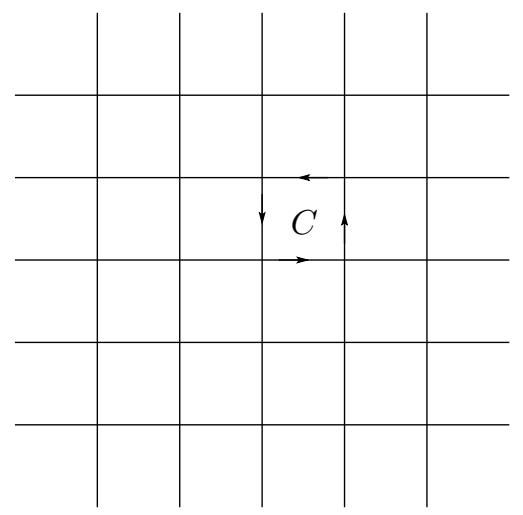

Figure 1: $W_{\infty}$

The action of the deck translations $F / F^{(1)} \cong \mathbb{Z} \times \mathbb{Z}$ is the obvious one where $x_{*}$ acts by horizontal translation and $y_{*}$ acts by vertical translation. Clearly $H_{1}\left(X_{1}\right)$ is an infintely generated abelian group but as a $\mathbb{Z}\left[x^{ \pm 1}, y^{ \pm 1}\right]$-module is cyclic, generated by the loop $C$ in Figure 1 which represents $x y x^{-1} y^{-1}$ under the identification $H_{1}\left(X_{1}\right) \cong F^{(1)} / F^{(2)}$. In fact $H_{1}\left(X_{1}\right)$ is a free $\mathbb{Z}\left[x^{ \pm 1}, y^{ \pm 1}\right]$ module generated by $C$. But $\mathcal{A}_{1}^{\mathbb{Z}}(K)=H_{1}\left(X_{1}\right)$ is a $\mathbb{Z}\left[G / G^{(2)}\right]-$ module and so far all we have discussed is the action of the subring $\mathbb{Z}\left[F / F^{(1)}\right]=\mathbb{Z}\left[G^{(1)} / G^{(2)}\right]$ because we have completely ignored the fact that $X_{0}$ itself has a $\mathbb{Z}$-action on it. In fact, since $1 \longrightarrow G^{(1)} / G^{(2)} \stackrel{i}{\longrightarrow} G / G^{(2)} \stackrel{\pi}{\longrightarrow} G / G^{(1)} \equiv \mathbb{Z} \longrightarrow 1$ is exact, any element of $G / G^{(2)}$ can be written as $g t^{m}$ for some $g \in G^{(1)} / G^{(2)}$ and $m \in \mathbb{Z}$ 
where $\pi(t)=1$. Thus we need only specify how $t_{*}$ acts on $H_{1}\left(X_{1}\right)$ to describe our module $\mathcal{A}_{1}^{\mathbb{Z}}(K)$. To see this action topologically, recall that, while $X_{0}$ is homotopy equivalent to $W$, a more precise description of it is as a countably infinite number of copies of $\Sigma \times[-1,1]$ where $\Sigma \times\{1\} \hookrightarrow(\Sigma \times[-1,1])_{i}$ is glued to $\Sigma \times\{-1\} \hookrightarrow(\Sigma \times[-1,1])_{i+1}$ by the homeomorphism $f$. Correspondingly, $X_{1}$ is homotopy equivalent to $\coprod_{i=-\infty}^{\infty}\left(W_{\infty} \times[-1,1]\right)$ glued together in just such a fashion by lifts of $f$ to $W_{\infty}$. Hence $t_{*}$ acts as $f_{*}$ acts on $H_{1}\left(X_{1}\right)=$ $F^{(1)} / F^{(2)}$. For example if $f_{*}(C)=f\left(x y x^{-1} y^{-1}\right)=w(x, y) C$ then $\mathcal{A}_{1}^{\mathbb{Z}}(K)$ is a cyclic module, generated by $C$, with relation $(t-w(x, y)) C=0$. Since $x y x^{-1} y^{-1}$ is represented by the circle $\partial \Sigma$, and since $f$ fixes this circle, in this case we have that $w(x, y)=1$ and $\mathcal{A}_{1}^{\mathbb{Z}}(K) \cong \mathbb{Z}\left[G / G^{(2)}\right] /(t-1) \mathbb{Z}\left[G / G^{(2)}\right]$. This is interesting because it has $t-1$ torsion represented by the longitude, whereas the classical Alexander module has no $t-1$ torsion. This reflects the fact that the longitude commutes with the meridian as well as the fact that the longitude, while trivial in $G / G^{(2)}$, is non-trivial in $G^{(2)} / G^{(3)} \equiv \mathcal{A}_{1}^{\mathbb{Z}}$.

Since the figure 8 knot is also a fibered genus 1 knot, its module has a similar form. But note that these modules are not isomorphic because they are modules over non-isomorphic rings (since the two knots do not have isomorphic classical Alexander modules $\left.G^{(1)} / G^{(2)}\right)$. This underscores that the higher Alexander modules $\mathcal{A}_{i}$ should only be used to distinguish knots with isomorphic $\mathcal{A}_{0}, \ldots, \mathcal{A}_{i-1}$.

The group of deck translations, $G / G^{(n)}$ of the $G^{(n)}$ cover of a knot complement is solvable but actually satisfies the following slightly stronger property.

Definition 2.5 A group $\Gamma$ is poly-(torsion-free abelian) (henceforth abbreviated PTFA) if it admits a normal series $\langle 1\rangle=G_{n} \triangleleft G_{n-1} \triangleleft \ldots \triangleleft G_{0}=\Gamma$ such that the factors $G_{i} / G_{i+1}$ are torsion-free abelian (Warning - in the group theory literature only a subnormal series is required).

This is a convenient class (as we shall see) because it is contained in the class of locally indicable groups [Str, Proposition 1.9] and hence $\mathbb{Z} \Gamma$ is an integral domain $\mathrm{Hig}$. Moreover it is contained in the class of amenable groups and thus $\mathbb{Z} \Gamma$ embeds in a classical quotient (skew) field [Do, Theorem 5.4].

It is easy to see that every PTFA group is solvable and torsion-free and although the converse is not quite true, every solvable group such that each $G^{(n)} / G^{(n+1)}$ is torsion-free, is PTFA. Every torsion-free nilpotent group is PTFA.

Consider a tower of regular covering spaces

$$
M_{n} \longrightarrow M_{n-1} \longrightarrow \ldots \longrightarrow M_{1} \longrightarrow M_{0}=M
$$


such that each $M_{i+1} \longrightarrow M_{i}$ has a torsion-free abelian group of deck translations and each $M_{i} \longrightarrow M$ is a regular cover. Then the group $\Gamma$ of deck translations of $M_{n} \longrightarrow M$ is PTFA and it is easy to see that such towers correspond precisely to normal series for such a group.

Example 2.6 If $G=\pi_{1}\left(S^{3} \backslash K\right)$ and $G^{(n)}$ is the $n^{\text {th }}$ term of the derived series then $G / G^{(n)}$ is PTFA since each $G^{(i)} / G^{(i+1)}$ is known to be torsion free Str]. Therefore taking iterated universal abelian covers of $S^{3}-K$ yields a PTFA tower as above. Hence the $n^{\text {th }}$ higher-order Alexander module generalizes the classical Alexander module in that the latter is the case of taking a single universal abelian covering space.

There is certainly more information to be found in modules obtained from other $\Gamma$-covers. For most of the proofs we can consider a general $\Gamma$-cover where $\Gamma$ is PTFA. Thus there are other families of subgroups which merit scrutiny, and are covered by most of the theorems to follow, but which will not be discussed in this paper. Primary among these is the lower central series of the commutator subgroup of $G$.

For a general 3-manifold with first Betti number equal to 1 (which we cover since it is no more difficult than a knot exterior) it is necessary to use the rational derived series to avoid zero divisors in the group ring:

Example 2.7 For any group $G$, the $n^{\text {th }}$ term of the rational derived series is defined by $G_{\mathbf{Q}}^{(0)}=G$ and $G_{\mathbf{Q}}^{(n)}=\left[G_{\mathbf{Q}}^{(n-1)}, G_{\mathbf{Q}}^{(n-1)}\right] \cdot N$ where $N=\{g \in$ $G_{\mathbf{Q}}^{(n-1)} \mid$ some non-zero power of $g$ lies in $\left.\left[G_{\mathbf{Q}}^{n-1}, G_{\mathbf{Q}}^{n-1}\right]\right\}$. It is easy to see that $G / G_{\mathbf{Q}}^{(n)}$ is PTFA. This corresponds to taking iterated universal torsion-free abelian covering spaces. For knot groups, $G_{\mathbf{Q}}^{(n)}=G^{(n)}$ Str].

Definition 2.8 If $M$ is an arbitrary connected CW-complex with fundamental group $G$, then the $n^{\text {th }}$ (integral) higher-order Alexander module, $\mathcal{A}_{n}^{\mathbb{Z}}(M)$, $n \geq 0$, of $M$ is $H_{1}\left(M_{n} ; \mathbb{Z}\right)\left(M_{n}\right.$ is the cover of $M$ with $\left.\pi_{1}\left(M_{n}\right)=G_{\mathbb{Q}}^{(n+1)}\right)$ considered as a right $\mathbb{Z}\left[G / G_{\mathbb{Q}}^{(n+1)}\right]$-module.

\section{More on the relationship of $\mathcal{A}_{n}^{\mathbb{Z}}(K)$ to $\pi_{1}\left(S^{3} \backslash K\right)$}

We have seen that if $H$ is any characteristic subgroup of $G$ then the isomorphism type of $H /[H, H]$, as a right module over $\mathbb{Z}[G / H]$, is an invariant of the isomorphism type of $G$. Moreover, $\mathcal{A}_{n}^{\mathbb{Z}}(K)$ has been defined as this module in 
the case $G=\pi_{1}\left(S^{3} \backslash K\right)$ and $H=G^{(n+1)}$. The following elementary observation clarifies this relationship. Its proof is left to the reader. One consequence will be that for any knot there exists a hyperbolic knot with isomorphic $\mathcal{A}_{n}^{\mathbb{Z}}$ for all $n$.

Proposition 2.9 Suppose $f: G \longrightarrow P$ is an epimorphism. Then $f$ induces isomorphisms $f_{n}: \mathcal{A}_{n}^{\mathbb{Z}}(G) \longrightarrow \mathcal{A}_{n}^{\mathbb{Z}}(P)$ for all $n \leq m$ if and only if the kernel of $f$ is contained in $G_{\mathbb{Q}}^{(m+2)}$. Hence $f$ induces such isomorphisms for all finite $n$ if and only if kernel $f \subset \bigcap_{n=1}^{\infty} G_{\mathbb{Q}}^{(n)}$.

Corollary 2.10 For any knot $K$, there is a hyperbolic knot $\widetilde{K}$ and a degree one map $f: S^{3} \backslash \widetilde{K} \longrightarrow S^{3} \backslash K$ (rel boundary) which induces isomorphisms $\mathcal{A}_{n}^{\mathbb{Z}}(\widetilde{K}) \longrightarrow \mathcal{A}_{n}^{\mathbb{Z}}(K)$ for all $n$.

Proof of Corollary 2.10 In fact it is known that $\widetilde{K}$ can be chosen so that the kernel of $f_{*}$ is a perfect group (or in other words that $f$ induces isomorphisms on homology with $\mathbb{Z}\left[\pi_{1}\left(S^{3} \backslash K\right)\right]$ coefficients). The first reference I know to this fact is by use of the "almost identical link imitations" of Akio Kawauchi Ka Theorem 2.1 and Corollary 2.2]. A more recent and elementary construction can be adopted from [BW] Section 4]. Any perfect subgroup is contained in its own commutator subgroup and hence, by induction, lies in every term of the derived series. An application of Proposition 2.9 finishes the proof.

Example 2.11 If $K^{\prime}$ is a knot and $K$ is a knot whose (classical) Alexander polynomial is 1 then $K^{\prime}$ and $K^{\prime} \# K$ have isomorphic higher-order modules since there is a degree one map $S^{3} \backslash\left(K^{\prime} \# K\right) \rightarrow S^{3} \backslash K^{\prime}$ which induces an epimorphism on $\pi_{1}$ whose kernel is $\pi_{1}\left(S^{3} \backslash K\right)^{(1)}$. The observation then follows from Proposition 2.9 and Example 2.3 .

\section{Properties of higher-order Alexander modules of knots: Torsion}

In this section we will show that higher-order Alexander modules have one key property in common with the classical Alexander module, namely they are torsion-modules. In Section 12 we define a linking pairing on these modules 
which generalizes the Blanchfield linking pairing on the Alexander module. All of the results of this section follow immediately from [COT1, Section 2] but a simpler proof of the main theorem is given here.

A right module $A$ over a ring $R$ is said to be a torsion module if, for any $a \in A$, there exists a non-zero-divisor $r \in R$ such that $a r=0$.

Our first goal is:

Theorem 3.1 The higher-order Alexander modules $\mathcal{A}_{n}^{\mathbb{Z}}(K)$ of a knot are torsion modules.

This is a consequence of the more general result which applies to any complex $X$ with $\pi_{1}(X)$ finitely-generated and $\beta_{1}(X)=1$ and any PTFA $\Gamma$ COT1, Proposition 2.11] but we shall give a different, self-contained proof (Proposition 3.10). The more general result will be used in later chapters to study general 3 -manifolds with $\beta_{1}=1$.

Suppose $\Gamma$ is a PTFA group. Then $\mathbb{Z} \Gamma$ has several convenient properties - it is an integral domain and it has a classical field of fractions. Details follow.

Recall that if $A$ is a commutative ring and $S$ is a subset closed under multiplication, one can construct the ring of fractions $A S^{-1}$ of elements $a s^{-1}$ which add and multiply as normal fractions. If $S=A-\{0\}$ and $A$ has no zero divisors, then $A S^{-1}$ is called the quotient field of $A$. However, if $A$ is non-commutative then $A S^{-1}$ does not always exist (and $A S^{-1}$ is not a priori isomorphic to $S^{-1} A$ ). It is known that if $S$ is a right divisor set then $A S^{-1}$ exists ( $[\mathrm{P}$ p. 146] or Ste, p. 52]). If $A$ has no zero divisors and $S=A-\{0\}$ is a right divisor set then $A$ is called an Ore domain. In this case $A S^{-1}$ is a skew field, called the classical right ring of quotients of $A$. We will often refer to this merely as the quotient field of $A$. A good reference for non-commutative rings of fractions is Chapter 2 of Ste. In this paper we will always use right rings of fractions.

Proposition 3.2 If $\Gamma$ is PTFA then $\mathbb{Q} \Gamma$ (and hence $\mathbb{Z} \Gamma$ ) is a right (and left) Ore domain; i.e. $\mathbb{Q} \Gamma$ embeds in its classical right ring of quotients $\mathcal{K}$, which is a skew field.

Proof For the fact (due to A.A. Bovdi) that $\mathbb{Z} \Gamma$ has no zero divisors see $\mathbb{P}$ pp. 591-592] or [Str p. 315]. As we have remarked, any PTFA group is solvable. It is a result of $J$. Lewin $[\mathrm{Lew}$ that for solvable groups such that $\mathbb{Q} \Gamma$ has no zero divisors, $\mathbb{Q} \Gamma$ is an Ore domain (see Lemma 3.6 iii p. 611 of $[\mathrm{P}]$ ). It follows that $\mathbb{Z} \Gamma$ is also an Ore domain. 
Remark 3.3 Skew fields share many of the key features of (commutative) fields. We shall need the following elementary facts about the right skew field of quotients $\mathcal{K}$. It is naturally a $\mathcal{K}-\mathcal{K}$-bimodule and a $\mathbb{Z} \Gamma-\mathbb{Z} \Gamma$-bimodule.

Fact $1 \mathcal{K}$ is flat as a left $\mathbb{Z} \Gamma$-module, i.e. $\cdot \otimes_{\mathbb{Z} \Gamma} \mathcal{K}$ is exact Ste, Proposition II.3.5].

Fact 2 Every module over $\mathcal{K}$ is a free module [Ste, Proposition I.2.3] and such modules have a well defined rank $\mathrm{rk}_{\mathcal{K}}$ which is additive on short exact sequences Co2, p. 48].

If $\mathcal{A}$ is a module over the Ore domain $\mathcal{R}$ then the rank of $\mathcal{A}$ denotes $\operatorname{rank}_{\mathcal{K}}\left(\mathcal{A}_{\mathcal{R}}\right.$ $\mathcal{K})$. $\mathcal{A}$ is a torsion module if and only if $\mathcal{A} \otimes_{\mathcal{R}} \mathcal{K}=0$ where $\mathcal{K}$ is the quotient field of $\mathcal{R}$, i.e. if and only if the rank of $\mathcal{A}$ is zero [Ste, II Corollary 3.3]. In general, the set of torsion elements of $A$ is a submodule which is characterized as the kernel of $\mathcal{A} \rightarrow \mathcal{A} \otimes_{\mathcal{R}} \mathcal{K}$. Note that if $\mathcal{A} \cong \mathcal{R}^{r} \oplus$ (torsion) then $\operatorname{rank} \mathcal{A}=r$.

Fact 3 If $\mathcal{C}$ is a non-negative finite chain complex of finitely generated free (right) $\mathbb{Z} \Gamma$-modules then the equivariant Euler characteristic, $\chi(\mathcal{C})$, given by $\sum_{i=0}^{\infty}(-1)^{i} \operatorname{rank} C_{i}$, is defined and equal to $\sum_{i=0}^{\infty}(-1)^{i} \operatorname{rank} H_{i}(\mathcal{C})$ and $\sum_{i=0}^{\infty}(-1)^{i} \operatorname{rank} H_{i}\left(\mathcal{C} \otimes_{\mathbb{Z} \Gamma} \mathcal{K}\right)$. This is an elementary consequence of Facts 1 and 2 .

There is another especially important property of PTFA groups (more generally of locally indicable groups) which should be viewed as a natural generalization of properties of the free abelian group. This is an algebraic generalization of the (non-obvious) fact that any infinite cyclic cover of a 2-complex with vanishing $H_{2}$ also has vanishing $H_{2}$ (see Proposition [3.8).

Proposition 3.4 (R. Strebel Str p. 305]) Suppose $\Gamma$ is a PTFA group and $R$ is a commutative ring. Any map between projective right $R \Gamma$-modules whose image under the functor $-\otimes_{R \Gamma} R$ is injective, is itself injective.

We can now offer a simple proof of Theorem 3.1

Proof of Theorem 3.1 The knot exterior has the homotopy type of a finite connected 2-complex $Y$ whose Euler characteristic is 0 . Let $\Gamma=G / G^{(n+1)}$ and let $\mathcal{C}=\left(0 \longrightarrow C_{2} \stackrel{\partial_{2}}{\longrightarrow} C_{1} \stackrel{\partial_{1}}{\longrightarrow} C_{0} \longrightarrow 0\right)$ be the free $\mathbb{Z} \Gamma$ cellular chain complex for $Y_{\Gamma}$ (the $\Gamma$-cover of $Y$ such that $\pi_{1}(Y)=G^{(n+1)}$ ) obtained by lifting the cell structure of $Y$. Then $\chi(\mathcal{C})=\chi(Y)=0$. It follows from Fact 3 that $\operatorname{rank} H_{2}\left(Y_{\Gamma}\right)-\operatorname{rank} H_{1}\left(Y_{\Gamma}\right)+\operatorname{rank} H_{0}\left(Y_{\Gamma}\right)=0$. Now note that $(\mathcal{C}, \partial)$ is 
sent, under the augmentation $\epsilon: \mathbb{Z} \Gamma \longrightarrow \mathbb{Z}$, to $\left(\mathcal{C} \otimes_{\mathbb{Z} \Gamma} \mathbb{Z}, \partial \otimes_{\mathbb{Z} \Gamma}\right.$ id $)$ which can be identified with the chain complex for the original cell structure on $Y$. Since $H_{2}(Y ; \mathbb{Z})=0, \partial_{2} \otimes$ id is injective. By Proposition 3.4 it follows that $\partial_{2}$ itself is injective, and hence that $H_{2}\left(Y_{\Gamma}\right)=0$.

Now we claim that $H_{0}\left(Y_{\Gamma}\right)$ is a torsion module. This is easy since $H_{0}\left(Y_{\Gamma}\right) \cong \mathbb{Z}$. If $H_{0}\left(Y_{\Gamma}\right)$ were not torsion then $1 \in \mathbb{Z}$ generates a free $\mathbb{Z} \Gamma$ submodule. Note that $\Gamma$ is not trivial since $G \neq G^{(1)}$. This is a contradiction since, as an abelian group, $\mathbb{Z} \Gamma$ is free on more than one generator and hence cannot be a subgroup of $\mathbb{Z}$.

Now that we have proved that the higher-order modules of a knot are torsion modules, we look at the homology of covering spaces in more detail and in a more abstract way. This point of view allows for greater generality and for more concise notation. Viewing homology of covering spaces as homology with twisted coefficients clarifies the calculations of the homology of induced covers over subspaces.

\section{Homology of PTFA covering spaces}

Suppose $X$ has the homotopy type of a connected CW-complex, $\Gamma$ is any group and $\phi: \pi_{1}\left(X, x_{0}\right) \longrightarrow \Gamma$ is a homomorphism. Let $X_{\Gamma}$ denote the regular $\Gamma-$ cover of $X$ associated to $\phi$ (by pulling back the universal cover of $B \Gamma$ viewed as a principal $\Gamma$-bundle). If $\phi$ is surjective then $X_{\Gamma}$ is merely the connected covering space $X$ associated to $\operatorname{Ker}(\phi)$. Then $X_{\Gamma}$ becomes a right $\Gamma$-set as follows. Choose a point $* \in p^{-1}\left(x_{0}\right)$. Given $\gamma \in \Gamma$, choose a loop $w$ in $X$ such that $\phi([w])=\gamma$. Let $\widetilde{w}$ be a lift of $w$ to $X_{\Gamma}$ such that $\widetilde{w}(0)=*$. Let $d_{w}$ be the unique covering translation such that $d_{w}(*)=\widetilde{w}(1)$. Then $\gamma$ acts on $X_{\Gamma}$ by $d_{w}$. This merely the "usual" left action [M2, Section 81]. However, for certain historical reasons we shall use the associated right action where $\gamma$ acts by $\left(d_{w}\right)^{-1}$. If $\phi$ is not surjective and we set $\pi=\operatorname{image}(\phi)$ then $X_{\Gamma}$ is a disjoint union of copies of the connected cover $X_{\pi}$ associated to $\operatorname{Ker}(\phi)$. The set of copies is in bijection with the set of right cosets $\Gamma / \pi$. In fact it is best to think of $p^{-1}\left(x_{0}\right)$ as being identified with $\Gamma$. Then $\Gamma$ acts on $p^{-1}\left(x_{0}\right)$ by right multiplication. If $\gamma \in \pi$, then $\gamma$ sends $*$ to the endpoint of the path $\widetilde{w}$ such that $\widetilde{w}(0)=*$ and $\phi([w])=\gamma^{-1}$. Hence $*$ and $(*) \gamma$ are in the same path component of $X_{\Gamma}$. If $\tau \in \Gamma$ is a non-trivial coset representative then $(*) \tau$ lies in a different path component than $*$. But the path $\widetilde{w}$, acted on by the deck translation corresponding to $\tau$, begins at $(*) \tau$ and ends at $(\widetilde{w}(1)) \tau=(*)(\gamma)(\tau)=(*)(\gamma \tau)$. Thus $(*) \tau$ and $(*) \tau^{\prime}$ lie in the same path component if and only if they lie in the same right coset $\pi \tau$ of $\Gamma / \pi$. 
For simplicity, the following are stated for the ring $\mathbb{Z}$, but also hold for $\mathbb{Q}$. Let $\mathcal{M}$ be a $\mathbb{Z} \Gamma$-bimodule (for us usually $\mathbb{Z} \Gamma, \mathcal{K}$, or a ring $\mathcal{R}$ such that $\mathbb{Z} \Gamma \subset$ $\mathcal{R} \subset \mathcal{K}$, or $\mathcal{K} / \mathcal{R})$. The following are often called the equivariant homology and cohomology of $X$.

Definition 3.5 Given $X, \phi, \mathcal{M}$ as above, let

$$
H_{*}(X ; \mathcal{M}) \equiv H_{*}\left(\mathcal{C}\left(X_{\Gamma} ; \mathbb{Z}\right) \otimes_{\mathbb{Z} \Gamma} \mathcal{M}\right)
$$

as a right $\mathbb{Z} \Gamma$ module, and $H^{*}(X ; \mathcal{M}) \equiv H_{*}\left(\operatorname{Hom}_{\mathbb{Z} \Gamma}\left(\mathcal{C}\left(X_{\Gamma} ; \mathbb{Z}\right), \mathcal{M}\right)\right)$ as a left $\mathbb{Z} \Gamma$-module.

These are also well-known to be isomorphic (respectively) to the homology (and cohomology) of $X$ with coefficient system induced by $\phi$ (see Theorems VI 3.4 and $3.4^{*}$ of $[\mathrm{W}]$ ). The advantage of this formulation is that it becomes clear that the surjectivity of $\phi$ is irrelevant.

\section{Remark 3.6}

(1) Note that $H_{*}(X ; \mathbb{Z} \Gamma)$ as in Definition 3.5 is merely $H_{*}\left(X_{\Gamma} ; \mathbb{Z}\right)$ as a right

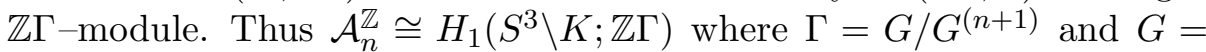
$\pi_{1}\left(S^{3} \backslash K\right)$. Moreover if $\mathcal{M}$ is flat as a left $\mathbb{Z} \Gamma$-module then $H_{*}(X ; \mathcal{M}) \cong$ $H_{*}\left(X_{\Gamma} ; \mathbb{Z}\right) \otimes_{\mathbb{Z} \Gamma} \mathcal{M}$. In particular this holds for $\mathcal{M}=\mathcal{K}$ by 3.3. Thus $H_{*}\left(X_{\Gamma}\right)=H_{*}(X ; \mathbb{Z} \Gamma)$ is a torsion module if and only if $H_{*}(X ; \mathcal{K})=$ $H_{*}\left(X_{\Gamma}\right) \otimes_{\mathbb{Z} \Gamma} \mathcal{K}=0$ by the remarks below 3.3 .

(2) Recall that if $X$ is a compact, oriented $n$-manifold then by Poincaré duality $H_{p}(X ; \mathcal{M})$ is isomorphic to $H^{n-p}(X, \partial X ; \mathcal{M})$ which is made into a right $\mathbb{Z} \Gamma$-module using the obvious involution on this group ring $[\mathrm{Wa}$.

(3) We also have a universal coefficient spectral sequence as in L3, Theorem 2.3]. This collapses to the usual Universal Coefficient Theorem for coefficients in a (noncommutative) principal ideal domain (in particular for the skew field $\mathcal{K})$. Hence $H^{n}(X ; \mathcal{K}) \cong \operatorname{Hom}_{\mathcal{K}}\left(H_{n}(X ; \mathcal{K}), \mathcal{K}\right)$. In this paper we only need the UCSS in these special cases where it coincides with the usual UCT.

We now restrict to the case that $\Gamma$ is a PTFA group and $\mathcal{K}$ is its (skew) field of quotients. We investigate $H_{0}, H_{1}$ and $H_{2}$ of spaces with coefficients in $\mathbb{Z} \Gamma$ or $\mathcal{K}$.

Proposition 3.7 Suppose $X$ is a connected $C W$ complex. If $\phi: \pi_{1}(X) \longrightarrow \Gamma$ is a non-trivial coefficient system then $H_{0}(X ; \mathcal{K})=0$ and $H_{0}(X ; \mathbb{Z} \Gamma)$ is a torsion module. 
Proof By W, p. 275] and [Br, p.34], $H_{0}(X ; \mathcal{K})$ is isomorphic to the cofixed set $\mathcal{K} / \mathcal{K} I$ where $I$ is the augmentation ideal of $\mathbb{Z} \pi_{1}(X)$ acting via $\pi_{1}(X) \longrightarrow$ $\Gamma \longrightarrow \mathcal{K}$. If $\phi$ is non-zero then this composition is non-zero and hence $I$ contains an element which acts as a unit. Hence $\mathcal{K} I=\mathcal{K}$.

The following lemma summarizes the basic topological application of Strebel's result (Proposition 3.4).

Proposition 3.8 Suppose $(Y, A)$ is a connected 2-complex with $H_{2}(Y, A ; \mathbb{Q})$ $\cong 0$ and suppose $\phi: \pi_{1}(Y) \longrightarrow \Gamma$ defines a coefficient system on $Y$ and $A$ where $\Gamma$ is a PTFA group. Then $H_{2}(Y, A ; \mathbb{Z} \Gamma)=0$, and so $H_{1}(A ; \mathbb{Z} \Gamma) \longrightarrow H_{1}(Y ; \mathbb{Z} \Gamma)$ is injective.

Proof Let $\mathcal{C}$ be the free $\mathbb{Z} \Gamma$ chain complex for the cellular structure on $\left(Y_{\Gamma}, A_{\Gamma}\right)$ (the $\Gamma$-cover of $Y$ ) obtained by lifting the cell structure of $(Y, A)$. It suffices to show $\partial_{2}: C_{2} \longrightarrow C_{1}$ is a monomorphism. By Proposition 3.4 this will follow from the injectivity of $\partial_{2} \otimes \mathrm{id}: C_{2} \otimes_{\mathbb{Z} \Gamma} \mathbb{Z} \longrightarrow C_{1} \otimes_{\mathbb{Z} \Gamma} \mathbb{Z}$. But this map can be canonically identified with the corresponding boundary map in the cellular chain complex of $(Y, A)$, which is injective since $H_{2}(Y, A ; \mathbb{Q}) \cong$ $H_{2}(Y, A ; \mathbb{Z}) \cong 0$.

The following lemma generalizes the key argument of the proof of Theorem 3.1

Lemma 3.9 Suppose $Y$ is a connected 2-complex with $H_{2}(Y ; \mathbb{Z})=0$ and $\phi: \pi_{1}(Y) \longrightarrow \Gamma$ is non-trivial. Then $H_{2}(Y ; \mathcal{K})=0$; and if $Y$ is a finite complex then $\operatorname{rk}_{\mathcal{K}} H_{1}(Y ; \mathcal{K})=\beta_{1}(Y)-1$.

Proof By Proposition $3.8 H_{2}(Y ; \mathbb{Z} \Gamma)=0$ and $H_{2}(Y ; \mathcal{K})=0$ by Remark 3.6.1. Since $\phi$ is non-trivial, Proposition 3.7 implies that $H_{0}(Y ; \mathcal{K})=0$. But by Fact 3 (as in the proof of Theorem 3.1) $\operatorname{rank}_{\mathcal{K}} H_{2}(Y ; \mathcal{K})-\operatorname{rank}_{\mathcal{K}} H_{1}(Y ; \mathcal{K})+$ $\operatorname{rank}_{\mathcal{K}} H_{0}(Y ; \mathcal{K})=1-\beta_{1}(Y)$ and the result follows.

Note that if $\beta_{1}(Y)=0$ then any homomorphism from $\pi_{1}(Y)$ to a PTFA group is necessarily the zero homomorphism.

Proposition 3.10 Suppose $\pi_{1}(X)$ is finitely-generated and $\phi: \pi_{1}(X) \longrightarrow \Gamma$ is non-trivial. Then

$$
\operatorname{rank}_{\mathcal{K}} H_{1}(X ; \mathbb{Z} \Gamma) \leq \beta_{1}(X)-1 .
$$

In particular, if $\beta_{1}(X)=1$ then $H_{1}(X ; \mathbb{Z} \Gamma)$ is a torsion module. 
Proof Since the first homology of a covering space of $X$ is functorially determined by $\pi_{1}(X)=G$, we can replace $X$ by a $K(G, 1)$. We will now construct an epimorphism $f: E \longrightarrow G$ from a group $E$ which has a very efficient presentation. Suppose $H_{1}(G) \cong \mathbb{Z}^{m} \times \mathbb{Z}_{n_{1}} \times \cdots \times \mathbb{Z}_{n_{k}}$. Then there is a finite generating set $\left\{g_{1}, \ldots, g_{m}, g_{m+1}, \ldots, g_{m+k}, \ldots \mid i \in I\right\}$ for $G$ such that $\left\{g_{1}, \ldots, g_{m+k}\right\}$ is a "basis" for $H_{1}(G)$ wherein if $i>m+k$ then $g_{i} \in[G, G]$ and if $m<i \leq m+k$ then $g_{i}^{n_{i}} \in[G, G]$. Consider variables $\left\{x_{j} \mid j \in I\right\}$. Hence for each $i$ there is a word $w_{i}\left(x_{1}, \ldots\right)$ in these variables such that $w_{i}$ lies in the commutator subgroup of the free group on $\left\{x_{j}\right\}$, and such that if $i>m+k$ then $g_{i}=w_{i}\left(g_{1}, \ldots\right)$ and if $m<i \leq m+k$ then $g_{i}^{n_{i}}=w_{i}\left(g_{1}, \ldots\right)$. Let $E$ have generators $\left\{x_{i} \mid i \in I\right\}$ and relations $\left\{x_{i}=w_{i} \mid i>m+k\right\}$ and $\left\{x_{i}^{n_{i}}=w_{i} \mid m<i \leq m+k\right\}$. The obvious epimorphism $f: E \longrightarrow G$ given by $f\left(x_{i}\right)=g_{i}$ is an $H_{1}$-isomorphism. The composition $\phi \circ f$ defines a $\Gamma$ covering space of $K(E, 1)$. Since $f$ is surjective we can build $K(G, 1)$ from $K(E, 1)$ by adjoining cells of dimensions at least 2 . Thus $H_{1}(G, E ; \mathbb{Z} \Gamma)=0$ because there are no relative 1 -cells and consequently $f_{*}: H_{1}(E ; \mathbb{Z} \Gamma) \longrightarrow H_{1}(G ; \mathbb{Z} \Gamma)$ is also surjective. Since $\mathcal{K}$ is a flat $\mathbb{Z} \Gamma$ module $f_{*}: H_{1}(E ; \mathcal{K}) \longrightarrow H_{1}(G ; \mathcal{K})$ is surjective. Thus $\operatorname{rank}_{\mathcal{K}} H_{1}(X ; \mathbb{Z} \Gamma)=\operatorname{rank}_{\mathcal{K}} H_{1}(X ; \mathcal{K}) \leq \operatorname{rank}_{\mathcal{K}} H_{1}(E ; \mathcal{K})$. Now note that $E=\pi_{1}(Y)$ where $Y$ is a connected, finite 2-complex (associated to the presentation) which has vanishing second homology. Again since $H_{1}$ is functorially determined by $\pi_{1}, H_{1}(E ; \mathcal{K}) \cong H_{1}(Y ; \mathcal{K})$. Lemma 3.9 above shows that $\operatorname{rank}_{\mathcal{K}} H_{1}(Y ; \mathcal{K})=\beta_{1}(Y)-1=\beta_{1}(E)-1=\beta_{1}(X)-1$ and the result follows.

Example 3.11 It is somewhat remarkable (and turns out to be crucially important) that the previous two results fail without the finiteness assumption. If Proposition 3.10 were true without the finiteness assumption, all of the inequalities of Theorem 5.4 would be equalities. Consider $E=\left\langle x, z_{i}\right| z_{i}=$ $\left.\left[z_{i+1}, x\right], i \in \mathbb{Z}\right\rangle$. This is the fundamental group of an (infinite) 2-complex with $H_{2}=0$. Note that $\beta_{1}(E)=1$. But the abelianization of $E^{(1)}$ has a presentation $\left\langle z_{i} \mid z_{i}=(1-x) z_{i+1}\right\rangle$ as a module over $\mathbb{Z}\left[x^{ \pm 1}\right]$ and thus has rank 1 , not $\beta_{1}(E)-1$ as would be predicted by Proposition 3.10

Corollary 3.12 Suppose $M$ is a compact, orientable, connected 3-manifold such that $\beta_{1}(M)=1$. Suppose $\phi: \pi_{1}(M) \longrightarrow \Gamma$ is a homomorphism that is non-trivial on abelianizations where $\Gamma$ is PTFA. Then $H_{*}(M, \partial M ; \mathcal{K}) \cong 0 \cong$ $H_{*}(M ; \mathcal{K})$.

Proof Propositions 3.7 and 3.10 imply $H_{0}(M ; \mathcal{K}) \cong H_{1}(M ; \mathcal{K}) \cong 0$. Since it is well known that the image of $H_{1}(\partial M ; \mathbb{Q}) \longrightarrow H_{1}(M ; \mathbb{Q})$ has one-half the rank of 
$H_{1}(\partial M ; \mathbb{Q}), \partial M$ must be either empty or a torus. Suppose the latter. Then this inclusion-induced map is surjective. Therefore the induced coefficient system $\phi \circ i_{*}: \pi_{1}(\partial M) \longrightarrow \Gamma$ is non-trivial since it is non-trivial on abelianizations. Thus $H_{0}(\partial M ; \mathcal{K})=0$ by Proposition [3.7 implying that $H_{1}(M, \partial M ; \mathcal{K})=0$. By Remark 3.6, $H_{2}(M ; \mathcal{K}) \cong H^{1}(M, \partial M ; \mathcal{K}) \cong \operatorname{Hom}\left(H_{1}(M, \partial M ; \mathcal{K}), \mathcal{K}\right) \cong 0$. Similarly $H_{3}(M ; \mathcal{K}) \cong 0$. Then $H_{*}(M ; \mathcal{K}) \cong 0 \Rightarrow H_{*}(M, \partial M ; \mathcal{K}) \cong 0$ by duality and the universal coefficient theorem.

Thus we have shown that the definition of the classical Alexander module, i.e. the torsion module associated to the first homology of the infinite cyclic cover of the knot complement, can be extended to higher-order Alexander modules $\mathcal{A}_{\Gamma}^{\mathbb{Z}}=H_{1}(M ; \mathbb{Z} \Gamma)$ which are $\mathbb{Z} \Gamma$ torsion modules associated to arbitrary PTFA covering spaces. Indeed, by Proposition 3.10, this is true for any finite complex with $\beta_{1}(M)=1$.

\section{Localized higher-order modules}

In studying the classical abelian invariants of knots, one usual studies not only the "integral" Alexander module, $H_{1}\left(S^{3} \backslash K ; \mathbb{Z}\left[t, t^{-1}\right]\right)$, but also the rational Alexander module $H_{1}\left(S^{3} \backslash K ; \mathbb{Q}\left[t, t^{-1}\right]\right)$. Even though some information is lost in this localization, $\mathbb{Q}\left[t, t^{-1}\right]$ is a principal ideal domain and one has a good classification theorem for finitely generated modules over a PID. Moreover the rational Alexander module is self-dual whereas the integral module is not [Go]. In considering the higher-order modules it is even more important to localize our rings $\mathbb{Z}\left[G / G^{(n)}\right]$ in order to define a higher-order "rational" Alexander module over a (non-commutative) PID. Here, significant information will be lost but this simplification is crucial to the definition of numerical invariants. Recall that an integral domain is a right (respectively left) PID if every right (respectively left) ideal is principal. A ring is a PID if it is both a left and right PID. The definition of the relevant PID's follows.

Let $G$ be a group with $\beta_{1}(G)=1$ and let $\Gamma_{n}=G / G_{\mathbb{Q}}^{(n+1)}$ (which is the same as the ordinary derived series for a knot group). Recall that the (integral) Alexander module was defined as $\mathcal{A}_{n}^{\mathbb{Z}}(G)=H_{1}\left(G ; \mathbb{Z} \Gamma_{n}\right)$ in Definition 2.1 and Definition 2.8. Below we will describe a PID $R_{n}$ such that $\mathbb{Q} \Gamma_{n} \subset R_{n} \subset \mathcal{K}_{n}$ and such that $R_{n}$ is a localization of $\mathbb{Q} \Gamma_{n}$, i.e. $R_{n}=\mathbb{Q} \Gamma_{n}\left(S^{-1}\right)$ where $S$ is a right divisor set in $\mathbb{Q} \Gamma_{n}$. Using this we define the "localized" derived modules. These will be analyzed further in Section 5. These PID's were crucial in our previous work COT1. 
Definition 4.1 The $n^{\text {th }}$ "localized" Alexander module of a knot $K$, or, simply, the $n^{\text {th }}$ Alexander module of $K$ is $\mathcal{A}_{n}(K)=H_{1}\left(S^{3} \backslash K ; R_{n}\right)$.

Proposition 4.2 The $n^{\text {th }}$ Alexander module is a finitely-generated torsion module over the PID $R_{n}$.

Proof Let $M_{n}$ denote the covering space of $M=S^{3} \backslash K$ with $\pi_{1}\left(M_{n}\right)=$ $G^{(n+1)}$. Then $\mathcal{A}_{n}(K)$ is the first homology of the chain complex $C_{*}\left(M_{n}\right) \otimes_{\mathbb{Z} \Gamma_{n}}$ $R_{n}$. This is a chain complex of finitely generated free $R_{n}$-modules since $M$ has the homotopy type of a finite complex and we can use the lift of this cell structure to $M_{n}$. Since a submodule of a finitely-generated free module over a PID is again a finitely-generated free module (【], Theorem 17), it follows that the homology groups are finitely generated.

Now we define the rings $R_{n}$ and show that they are PID's by proving that they are isomorphic to skew Laurent polynomial rings $\mathbb{K}_{n}\left[t^{ \pm 1}\right]$ over a skew field $\mathbb{K}_{n}$. This makes the analogy to the classical rational Alexander module even stronger.

Before defining $R_{n}$ in general, we do so in a simple example.

Example 4.3 We continue with Example 2.4 where $G=\pi_{1}\left(S^{3} \backslash K\right)$ and $K$ is a trefoil knot. We illustrate the structure of $\mathbb{Z}\left[G / G^{(2)}\right]=\mathbb{Z} \Gamma_{1}$ as a skew Laurent polynomial ring in one variable with coefficients in $\mathbb{Z}\left[G^{(1)} / G^{(2)}\right]$. Recall that since the trefoil knot is fibered, $G^{(1)} / G^{(2)} \cong F / F^{(1)} \cong \mathbb{Z} \times \mathbb{Z}$ generated by $\{x, y\}$. Hence $\mathbb{Z}\left[G^{(1)} / G^{(2)}\right]$ is merely the (commutative) Laurent polynomial $\operatorname{ring} \mathbb{Z}\left[x^{ \pm 1}, y^{ \pm 1}\right]$. If we choose, say, a meridian $\mu \in G / G^{(2)}$ then $G / G^{(2)}$ is a semi-direct product $G^{(1)} / G^{(2)} \rtimes \mathbb{Z}$ and any element of $G / G^{(2)}$ has a unique representative $\mu^{m} g$ for some $m \in \mathbb{Z}$ and $g \in G^{(1)} / G^{(2)}$, i.e. $\mu^{m} x^{p} y^{q}$ for some integers $m, p, q$. Thus any element of $\mathbb{Z}\left[G / G^{(2)}\right]$ has a canonical representation of the form $\sum_{m=-\infty}^{\infty} \mu^{m} p_{m}(x, y)$ where $p_{m}(x, y) \in \mathbb{Z}\left[x^{ \pm 1}, y^{ \pm 1}\right]$. Hence $\mathbb{Z}\left[G / G^{(2)}\right]$ can be identified with the Laurent polynomial ring in one variable $\mu$ (or $t$ for historical significance) with coefficients in the Laurent polynomial ring $\mathbb{Z}\left[x^{ \pm 1}, y^{ \pm 1}\right]$. Observe that the product of 2 elements in canonical form is not in canonical form. However, for example, $\left(x^{p} y^{q}\right) \cdot \mu=\mu\left(\mu^{-1} x^{p} y^{q} \mu\right)=\mu\left(\left(x^{p} y^{q}\right) \mu_{*}\right)$. Hence this is not a true polynomial ring, rather the multiplication is twisted by the automorphism $\mu_{*}$ of $\mathbb{Z}\left[G^{(1)} / G^{(2)}\right]$ induced by conjugation $g \rightarrow \mu^{-1} g \mu$ (the action of the generator $t \in \mathbb{Z}$ in the semi-direct product structure). The action $\mu_{*}\left(\right.$ or $\left.t_{*}\right)$ is merely the action of $t$ on the Alexander module of the trefoil $\mathbb{Z}\left[t, t^{-1}\right] / t^{2}-t+1 \cong \mathbb{Z} \times \mathbb{Z}$ with basis $\{x, y\}$. 
Moreover this skew polynomial ring $\mathbb{Z}\left[G^{(1)} / G^{(2)}\right]\left[t^{ \pm 1}\right]$ embeds in the ring $R_{1}=$ $\mathbb{K}_{1}\left[t^{ \pm 1}\right]$, where $\mathbb{K}_{1}$ is the quotient field of the coefficient ring $\mathbb{Z}\left[x^{ \pm 1}, y^{ \pm 1}\right]$ (in this case the (commutative) field of rational functions in the 2 commuting variables $x$ and $y$ ). Thus $\mathbb{Z}\left[G / G^{(2)}\right]$ embeds in this (noncommutative) PID $R_{1}$ (this is proved below) that also has the structure of a skew Laurent polynomial ring over a field. Note that, under this embedding, the subring $\mathbb{Z}\left[G^{(1)} / G^{(2)}\right]$ is sent into the subring of polynomials of degree 0 , i.e. $\mathbb{K}_{1}$ and this embedding is just the canonical embedding of a commutative ring into its quotient field (and is thus independent of the choice of $\mu$ !).

Now we define $R_{n}$ in general. Let $\widetilde{G}_{n}, n \geq 1$, be the kernel of the map $\pi: G / G_{\mathbb{Q}}^{(n)} \longrightarrow G / G_{\mathbb{Q}}^{(1)}$ (the latter is infinite cyclic by the hypothesis that $\beta_{1}(G)=1$. For the important case that $G$ is a knot group, $\widetilde{G}_{n}$ is the commutator subgroup modulo the $n^{\text {th }}$ derived subgroup. Since $G / G_{\mathbb{Q}}^{(n)}$ is PTFA by Example 2.7 , the subgroup $\widetilde{G}_{n}$ is also PTFA. Thus $\mathbb{Z}\left[\widetilde{G}_{n}\right]$ is an Ore domain by Proposition 3.2 Let $S_{n}=\mathbb{Z}\left[\widetilde{G}_{n+1}\right]-\{0\}, n \geq 0$, a subset of $\mathbb{Z} \Gamma_{n}=\mathbb{Z}\left[G / G_{\mathbb{Q}}^{(n+1)}\right]$. By $[\mathrm{P}$, p. 609$] S_{n}$ is a right divisor set of $\mathbb{Z} \Gamma_{n}$ and we set $R_{n}=\left(\mathbb{Z} \Gamma_{n}\right)\left(S_{n}\right)^{-1}$. Hence $\mathbb{Z} \Gamma_{n} \subseteq R_{n} \subseteq \mathcal{K}_{n}$. Note that $S_{0}=\mathbb{Z}-\{0\}$ so $R_{0}=\mathbb{Q}[J]$ where $J$ is the infinite cyclic group $G / G_{\mathbb{Q}}^{(1)}$, agreeing with the classical case. By Proposition II.3.5 [Ste] we have the following.

Proposition 4.4 $R_{n}$ is a flat left $\mathbb{Z} \Gamma_{n}$-module so $\mathcal{A}_{n} \cong \mathcal{A}_{n}^{\mathbb{Z}} \otimes_{\mathbb{Z} \Gamma_{n}} R_{n}$. Moreover $\mathcal{K}_{n}$ is a flat $R_{n}$-module so $\mathcal{A}_{n} \otimes_{R_{n}} \mathcal{K}_{n}=H_{1}\left(M ; \mathcal{K}_{n}\right)$.

Now we establish that the $R_{n}$ are PID's. Consider the short exact sequence $1 \longrightarrow \widetilde{G} \longrightarrow G / G_{\mathbb{Q}}^{(n)} \stackrel{\pi}{\rightarrow} \mathbb{Z} \longrightarrow 1$ where $\pi$ is induced by abelianization and $\widetilde{G}$ is the kernel of $\pi$. Note that there are precisely two such epimorphisms $\pi$. If we choose $\mu \in G / G_{\mathbb{Q}}^{(n)}$ which generates the torsion-free part of the abelianization then $\pi$ is canonical (take $\pi(\mu)=1$ ) and has a canonical splitting $(1 \stackrel{s}{\rightarrow} \mu)$. Now note that any element of $\mathbb{Q}\left[G / G_{\mathbb{Q}}^{(n)}\right]$ has a unique expression of the form $\gamma=\mu^{-m} a_{-m}+\cdots+a_{0}+\cdots+\mu^{k} a_{k}$ where $a_{i} \in \mathbb{Q} \widetilde{G}$ ( $a_{-m}$ and $a_{k}$ not zero unless $\gamma=0)$. Thus $\mathbb{Q}\left[G / G_{\mathbb{Q}}^{(n)}\right]$ is canonically isomorphic to the skew Laurent polynomial ring, $\mathbb{Q} \widetilde{G}\left[t^{ \pm 1}\right]$, in one variable with coefficients in $\mathbb{Q} \widetilde{G}$. Recall that the latter is the ring consisting of expressions $t^{-m} a_{-m}+\cdots+t^{k} a_{k}, a_{i} \in \mathbb{Q} \widetilde{G}$ which add as ordinary polynomials but where multiplication is twisted by an automorphism $\alpha: \mathbb{Q} \widetilde{G} \longrightarrow \mathbb{Q} \widetilde{G}$ so that if $a \in \mathbb{Q} \widetilde{G}$ then $t^{i} a \cdot t=t^{i+1} \alpha(a)$. The automorphism in our case is induced by the automorphism of $\widetilde{G}$ given by 
conjugation by $\mu$. The twisted multiplication is evident in $\widetilde{G}$ since $\mu^{i} a \cdot \mu=$ $\mu^{i} \mu\left(\mu^{-1} a \mu\right)=\mu^{i+1} \alpha(a)$.

Since $\widetilde{G}$ is a subgroup of a PTFA group, it also is PTFA and so $\mathbb{Z} \widetilde{G}$ admits a (right) skew field of fractions $\mathbb{K}$ into which it embeds. This is also written $(\mathbb{Z} \widetilde{G})(\mathbb{Z} \widetilde{G})^{-1}$ meaning that all the non-zero elements of $\mathbb{Z} \widetilde{G}$ are inverted. It follows that $\mathbb{Z}\left[G / G_{\mathbb{Q}}^{(n)}\right](\mathbb{Z} \widetilde{G})^{-1}$ is canonically identified with the skew polynomial ring $\mathbb{K}\left[t^{ \pm 1}\right]$ with coefficients in the skew field $\mathbb{K}$ (see COT1, Proposition 3.2] for more details). The following is well known (see Chapter 3 of [J] or Prop. 2.1.1 of [Co1] ).

Proposition 4.5 A skew polynomial ring $\mathbb{K}\left[t^{ \pm 1}\right]$ over a division ring $\mathbb{K}$ is a right (and left) PID.

Proof One first checks that there is a well-defined degree function on any skew Laurent polynomial ring (over a domain) where $\operatorname{deg}\left(t^{-m} a_{-m}+\cdots+t^{k} a_{k}\right)=m+$ $k$ and that this degree function is additive under multiplication of polynomials. Then one verifies that there is a division algorithm such that if $\operatorname{deg}(q(t)) \geq$ $\operatorname{deg}(p(t))$ then $q(t)=p(t) s(t)+r(t)$ where $\operatorname{deg}(r(t))<\operatorname{deg}(p(t))$. Finally, if $I$ is any non-zero right ideal, choose $p \in I$ of minimal degree. For any $q \in I$, $q=p s+r$ where, by minimality, $r=0$. Hence $I$ is principal. Thus $\mathbb{K}\left[t^{ \pm 1}\right]$ is a right PID. The proof that it is a left PID is identical.

Proposition 4.6 For $n \geq 0$ let $R_{n}$ denote the $\operatorname{ring} \mathbb{Z}\left[G / G^{(n+1)}\right](\mathbb{Z} \widetilde{G})^{-1}$. This can be identified with the PID $\mathbb{K}_{n}\left[t^{ \pm 1}\right]$ where $\mathbb{K}_{n}$ is the quotient field of $\mathbb{Z} \widetilde{G}$ $\left(1 \longrightarrow \widetilde{G} \longrightarrow G / G^{(n+1)} \stackrel{\pi}{\longrightarrow} \mathbb{Z} \longrightarrow 1\right)$.

Of course the isomorphism type of $\mathcal{A}_{n}(K)$ is still purely a function of the isomorphism type of the group $G$ of the knot since $\mathcal{A}_{n}(K)=G^{(n+1)} / G^{(n+2)} \otimes$ $R_{n}$. However, when viewed as a module over $\mathbb{K}_{n}\left[t^{ \pm 1}\right]$, it is also dependent on a choice of the meridional element $\mu$.

\section{Non-triviality}

We now show that the higher-order Alexander modules are never trivial except when $K$ is a knot with Alexander polynomial 1. The following results generalize Proposition 3.10 and Lemma 3.9 .

Corollary 4.7 If $X$ is a (possibly infinite) 2-complex with $H_{2}(X ; \mathbb{Q})=0$ and $\phi: \pi_{1}(X) \longrightarrow \Gamma$ is a PTFA coefficient system then $\operatorname{rank}\left(H_{1}(X ; \mathbb{Z} \Gamma)\right) \geq$ $\beta_{1}(X)-1$. 
Corollary 4.8 If $K$ is a knot whose Alexander polynomial $\Delta_{0}$ is not 1 , then the derived series of $G=\pi_{1}\left(S^{3} \backslash K\right)$ does not stabilize at finite $n$, i.e. $G^{(n)} / G^{(n+1)} \neq 0$. Hence the derived module $\mathcal{A}_{n}^{\mathbb{Z}}(K)$ is non-trivial for any $n$. Moreover, if $n>0, \mathcal{A}_{n}(K)$ (viewed as a $\mathbb{K}_{n}\left[t^{ \pm 1}\right]$ module) has rank at least $\operatorname{deg}\left(\Delta_{0}(K)\right)-1$ as a $\mathbb{K}_{n}$-module and hence is an infinite dimensional $\mathbb{Q}$ vector space.

The first part of the Corollary has been independently established by S.K. Roushon $\mathrm{Ru}$.

Proposition $3.8 \Rightarrow$ Corollary 4.7 First consider the case that $\beta_{1}(X)$ is finite. Consider the case of Proposition 3.8 where $A$ is a wedge of $\beta_{1}(X)$ circles and $i: A \longrightarrow X$ is chosen to be a monomorphism on $H_{1}\left(\_; \mathbb{Q}\right)$. Then $\operatorname{rank}\left(H_{1}(X ; \mathbb{Z} \Gamma)\right)$ is at least $\operatorname{rank}\left(H_{1}(A ; \mathbb{Z} \Gamma)\right)$ which is $\beta_{1}(X)-1$ by Lemma 3.9 Now if $\beta_{1}(X)$ is infinite, apply the above argument for a wedge of $n$ circles where $n$ is arbitrary.

Proposition $3.8 \Rightarrow$ Corollary 4.8 Let $X$ be the infinite cyclic cover of $S^{3} \backslash K$, and let $\widetilde{G}=\pi_{1}(X) / \pi_{1}(X)^{(n)}=G^{(1)} / G^{(n+1)}$ as in Proposition 4.6. If $\Delta_{0} \neq 1$ then $\operatorname{deg}\left(\Delta_{0}\right)=\beta_{1}(X) \geq 2$. Applying Corollary 4.7 we get that $H_{1}(X ; \mathbb{Z} \widetilde{G})$ has rank at least $\beta_{1}(X)-1$. But $H_{1}(X ; \mathbb{Z} \widetilde{G})$ can be interpreted as the first homology of the $\widetilde{G}$-cover of $X$, as a $\mathbb{Z} \widetilde{G}$ module. This covering space has $\pi_{1}$ equal to $G^{(n+1)}$. Since the $\widetilde{G}$ cover of $X$ is the same as the cover of $S^{3} \backslash K$ induced by $G \longrightarrow G / G^{(n+1)}, H_{1}(X ; \mathbb{Z} \widetilde{G}) \cong H_{1}\left(S^{3} \backslash K ; \mathbb{Z}\left[G / G^{(n+1)}\right]\right) \equiv \mathcal{A}_{n}^{\mathbb{Z}}(K)$ as $\mathbb{Z} \widetilde{G}$-modules. Now, since $\mathcal{A}_{n}^{\mathbb{Z}}$ has rank at least $\beta_{1}(X)-1$ as a $\mathbb{Z} \widetilde{G}$-module, $\mathcal{A}_{n}$ has rank at least $\beta_{1}(X)-1$ as a $\mathbb{K}_{n}$ module since the latter is the definition of the former. It follows that $G^{(n+1)} / G^{(n+2)}$ is non-trivial (and hence infinite) for $n \geq 0$. If $n>0$ it follows that $\widetilde{G}$ is an infinite group. In this case $\mathbb{Q} \widetilde{G}$ and hence $\mathbb{K}_{n}$ are infinitely generated vector spaces.

\section{$5 \quad$ Higher order Alexander polynomials}

In this section we further analyze the localized Alexander modules $\mathcal{A}_{n}(K)$ that were defined in Section 4 as right modules over the skew Laurent polynomial rings $R_{n} \cong \mathbb{K}_{n}\left[t^{ \pm 1}\right]$. We define higher-order "Alexander polynomials" $\Delta_{n}(K)$ and show that their degrees $\delta_{n}(K)$ are integral invariants of the knot. We prove that $\delta_{0}, \delta_{1}+1, \delta_{2}+1, \ldots$ is a non-decreasing sequence for any knot. In later sections we will see that the $\delta_{n}$ are powerful knot invariants with applications 
to genus and fibering questions. The higher-order Alexander polynomials bear further study.

Recall that it has already been established that $\mathcal{A}_{n}(K)$ is a finitely-generated torsion right $R_{n}$ module where $R_{n}$ is a PID. The following generalization of the standard theorem for commutative PIDs is well known (see Theorem 2.4 p. 494 of $[\mathrm{Co} 2$ ).

Theorem 5.1 Let $R$ be a principal ideal domain. Then any finitely generated torsion right $R$-module $M$ is a direct sum of cyclic modules

$$
M \cong R / e_{1} R \oplus \cdots \oplus R / e_{r} R
$$

where $e_{i}$ is a total divisor of $e_{i+1}$ and this condition determines the $e_{i}$ up to similarity.

Here $a$ is similar to $b$ if $R / a R \cong R / b R$ (p. 27 Co1]). For the definition of total divisor, the reader is referred to Chapter 8 of Co2. This complication is usually unnecessary because a finitely generated torsion module over a simple PID is cyclic (pp. 495-496 Co2])!! For $n>0, R_{n}$ is almost always a simple ring, but since this fact will not be used in this paper, we do not justify it.

Definition 5.2 For any knot $K$ and any integer $n \geq 0,\left\{e_{1}(K), \ldots, e_{r}(K)\right\}$ are the elements of the PID $R_{n}$, well-defined up to similarity, associated to the canonical decomposition of $\mathcal{A}_{n}(K)$. Let $\Delta_{n}(K)$, the $n^{\text {th }}$ order Alexander polynomial of $K$, be the product of these elements, viewed as an element of $\mathbb{K}_{n}\left[t^{ \pm 1}\right]$ (for $n=0$ this is the classical Alexander polynomial).

The polynomial $\Delta_{n}(K)$, as an element of $R_{n}$, is also well-defined up to similarity (a non-obvious fact that we will not use). However as an element of $\mathbb{K}_{n}\left[t^{ \pm 1}\right]$ it acquires additional ambiguity because a splitting of $G \rightarrow \mathbb{Z}$ was used to choose an isomorphism between $R_{n}$ and $\mathbb{K}_{n}\left[t^{ \pm 1}\right]$. Alternatively, using a square presentation matrix for $\mathcal{A}_{n}(K)$ (see the next section), one can associate an element of $K_{1}\left(R_{n}\right)$ and, using the Dieudonné determinant, recover $\Delta_{n}(K)$ as an element of $U /[U, U]$ where $U$ is the group of units of the quotient field of $R_{n}$. Since similarity is not well-understood in a noncommutative ring (being much more difficult than merely identifying when elements differ by units), we have not yet been able to make effective use of the higher-order Alexander polynomials except for their degrees, which turn out to be perfectly well-defined integral invariants, as we now explain. 
Definition 5.3 For any knot $K$ and any integer $n \geq 0$, the degree of the $n^{\text {th }}$ order Alexander polynomial, denoted $\delta_{n}(K)$ is an invariant of $K$. It can be defined in any of the following equivalent ways:

1) the degree of $\Delta_{n}(K)$

2) the sum of the degrees of $e_{i}(K) \in R_{n} \cong \mathbb{K}_{n}\left[t^{ \pm 1}\right]$

3) the rank of $\mathcal{A}_{n}(K)$ as a module over $\mathbb{K}_{n}$

4) the rank of $G^{(n+1)} / G^{(n+2)} \otimes_{\mathbb{Z} \Gamma_{n}} R_{n}$ as a module over the subring $\mathbb{Z} \widetilde{G} \subseteq$ $\mathbb{Z} \Gamma_{n}$

5) the rank of $G^{(n+1)} / G^{(n+2)}$ as a module over the subring $\mathbb{Z}\left[G^{(1)} / G^{(n+1)}\right] \subset$ $\mathbb{Z}\left[G / G^{(n+1)}\right]$.

Proof of Definition 5.3 Definitions 4 and 5 are independent of choices since there $R_{n}$ has not been specifically identified with the polynomial ring $\mathbb{K}_{n}$. To see that Definition 3 is the same as 4, consider Definition 2.1 and Proposition 4.4. Also note that the identification of $\mathbb{Z}\left[G / G^{(n+1)}\right]$ with the skew polynomial ring $\mathbb{Z} \widetilde{G}\left[t^{ \pm 1}\right]$, carries the subring $\mathbb{Z} \widetilde{G}$ (independent of splitting) to the ring of elements of degree zero. Thus under any identification of $R_{n}=\mathbb{Z}\left[G / G^{(n+1)}\right]$ $(\mathbb{Z} \widetilde{G}-\{0\})^{-1}$ with $\mathbb{K}_{n}\left[t^{ \pm 1}\right]$, the quotient field $\mathbb{Z} \widetilde{G}(\mathbb{Z} \widetilde{G}-\{0\})^{-1}$ is carried (independent of splitting) to $\mathbb{K}_{n}$, viewed as the subfield of elements of degree zero. From Definition 3 and Theorem [5.1 , one sees that these ranks are finite because the rank of $\mathbb{K}_{n}\left[t^{ \pm 1}\right] / p(t) \mathbb{K}_{n}\left[t^{ \pm 1}\right]$ is easily seen to be the degree of $p(t)$. The equivalence of Definitions 1 and 2 then follows trivially. To see that 4 and 5 are equivalent, one must show that $\mathcal{A}_{n}^{\mathbb{Z}} \otimes_{\mathbb{Z}\left[G / G^{(n+1)}\right]} \mathbb{K}_{n}\left[t^{ \pm 1}\right]$ as a $\mathbb{K}_{n}$-module is merely $\mathcal{A}_{n}^{\mathbb{Z}} \otimes_{\mathbb{Z} \widetilde{G}} \mathbb{K}_{n}$. This is left to the reader.

We can establish one interesting property of the $\delta_{n}$, namely that for any $K$ they form a non-decreasing sequence. This theorem says that the derived series of the fundamental group of a knot complement (more generally of certain 2complexes) cannot stabilize unless $\delta_{0}=1$ (see Corollary 4.8). Moreover in some sense the "size" of the successive quotients $G^{(n)} / G^{(n+1)}$ is non-decreasing.

Theorem 5.4 If $K$ is a knot then $\delta_{0}(K) \leq \delta_{1}(K)+1 \leq \delta_{2}(K)+1 \leq \cdots \leq$ $\delta_{n}(K)+1$.

Proof First we show $\delta_{1} \geq \delta_{0}-1$. Let $X$ be the infinite cyclic cover of $S^{3} \backslash K$ and $G=\pi_{1}\left(S^{3} \backslash K\right)$. Note that $\beta_{1}(X)=\operatorname{rank}_{\mathbb{Q}} H_{1}\left(S^{3} \backslash K ; \mathbb{Q}\left[t, t^{-1}\right]\right)=\delta_{0}$, and $\delta_{1}=\operatorname{rank}_{\mathbb{K}_{1}} H_{1}\left(S^{3} \backslash K ; \mathbb{K}_{1}\left[t^{ \pm 1}\right]\right)=\operatorname{rank} H_{1}\left(X ; \mathbb{Z}\left[G^{(1)} / G^{(2)}\right]\right)$ by Definition 5.3 
The latter, by Corollary 4.7 is at least $\beta_{1}(X)-1\left(\right.$ since $\left.H_{2}(X ; \mathbb{Q})=0\right)$ and we are done.

Now it will suffice to show $\delta_{n} \geq \delta_{n-1}$ if $n \geq 2$. Let $X_{n}$ be the covering space of $S^{3} \backslash K$ with fundamental group $G^{(n+1)}$ so $X_{0}=X$. Then $X_{n-1}$ is a covering space of $X$ with $G^{(1)} / G^{(n)}$ as deck translations. Choose a wedge of $\delta_{0}$ circles $A_{0} \rightarrow X$ giving an isomorphism on $H_{1}\left(\_; \mathbb{Q}\right)$. Let $\widetilde{A}_{0} \stackrel{i}{\rightarrow} X_{n-1}$ be the induced cover and corresponding inclusion. By Proposition [3.8, $i_{*}$ is a monomorphism on $H_{1}$. Since $n \geq 2, \operatorname{rank}_{\mathbb{Z}\left[G^{(1)} / G^{(n)}\right]} H_{1}\left(A_{0} ; \mathbb{Z}\left[G^{(1)} / G^{(n)}\right]\right)$ is precisely $\beta_{1}\left(A_{0}\right)-1=\delta_{0}-1$ by Lemma 3.9 (here we assume $\delta_{0}>0$ since if $\delta_{0}=0$ then $\delta_{i}=0$ and the theorem holds). Choose a subset of image $i_{*}$ with cardinality $\delta_{0}-1$ that is $\mathbb{Z}\left[G^{(1)} / G^{(n)}\right]$-linearly independent in $H_{1}\left(X_{n-1}\right)$. It is not difficult to show that, in a module over an Ore domain, any linearly independent set can be extended to a maximal linearly independent set, i.e. whose cardinality is equal to the rank of the module. Hence if $\delta_{n-1}$ (which equals the $\mathbb{Z}\left[G^{(1)} / G^{(n)}\right]$-rank of $\left.H_{1}\left(X_{n-1}\right)\right)$ exceeds $\delta_{0}-1$, then there is a set of $e=\delta_{n-1}-\left(\delta_{0}-1\right)$ circles and a map $\widetilde{f}: A_{e} \rightarrow X_{n-1}$ of a wedge of $e$ circles, such that the free submodule generated by these circles captures the "excess rank." Let $f=\pi \circ \tilde{f}: A_{e} \rightarrow X$. Then the map $A=A_{0} \vee A_{e} \longrightarrow X$ induces a monomorphism on $H_{1}\left(\_; \mathbb{Z}\left[G^{(1)} / G^{(n)}\right]\right)$ by construction. Another way of saying this is that the induced map on $G^{(1)} / G^{(n)}$-covers $A_{n-1} \rightarrow X_{n-1}$ is injective on $H_{1}\left(\_; \mathbb{Z}\right)$ where $A_{n-1}$ is the induced cover of $A$. Since $H_{2}(X ; \mathbb{Z})=0$, it follows from Lemma 3.9 that $H_{2}\left(X_{n-1} ; \mathbb{Z}\right)=0$. Hence $\left(X_{n-1}, A_{n-1}\right)$ is a relative 2 complex that satisfies the conditions of Proposition 3.8, with $\Gamma=G^{(n)} / G^{(n+1)}$. It follows that $H_{1}\left(A_{n-1} ; \mathbb{Z} \Gamma\right) \stackrel{i_{*}}{\rightarrow} H_{1}\left(X_{n-1} ; \mathbb{Z} \Gamma\right)$ is injective. But this is the same as the map induced by $i: A \rightarrow X$ on $H_{1}\left(\ldots ; \mathbb{Z}\left[G^{(1)} / G^{(n+1)}\right]\right)$. Thus $\delta_{n}=$ $\operatorname{rank} H_{1}\left(X ; \mathbb{Z}\left[G^{(1)} / G^{(n+1)}\right]\right)$ is at least the rank of $H_{1}\left(A ; \mathbb{Z}\left[G^{(1)} / G^{(n+1)}\right]\right)$. Since $A$ is a wedge of $e+\delta_{0}=\delta_{n-1}+1$ circles and $n \geq 2$, this latter rank is precisely $\delta_{n-1}$ by Lemma [3.9] Hence $\delta_{n} \geq \delta_{n-1}$ as claimed.

Question Is there a knot $K$ and some $n>0$ for which $\delta_{n}(K)$ is a non-zero even integer?

If not then a complete realization theorem for the $\delta_{i}$ can be derived from the techniques of Section 7 .

\section{Presentation of $\mathcal{A}_{n}$ from a Seifert surface}

Suppose $M$ is a knot exterior, or more generally a compact, connected, oriented 3 -manifold with $\beta_{1}=1$ that is either closed or whose boundary is a 
torus. Suppose $\pm V$ is a compact, connected, oriented surface which generates $H_{2}(M, \partial M)$. In the case of a knot exterior, the orientation on the knot can be used to fix the orientation of $V$, and $V$ can be chosen to be a Seifert surface of $K$. The classical Alexander module of $K$ can be calculated from a presentation matrix which is obtained by pushing certain loops in $V$ into $S^{3} \backslash V$. Here we show that there is a finite presentation of $\mathcal{A}_{n}(K)$ obtained in a similar fashion from $V$.

Let $Y=M-(V \times(-1,1))$ and denote by $i_{+}$and $i_{-}$the two inclusions $V \longrightarrow V \times\{ \pm 1\} \longrightarrow \partial Y \subset Y$. Recall from Definition 4.1 and Proposition 4.6 that $\mathcal{A}_{n}(M) \cong H_{1}\left(M ; \mathbb{K}_{n}\left[t^{ \pm 1}\right]\right)$ where an isomorphism is fixed by choosing a circle $u$ dual to $V$ (an oriented meridian in the case that $M=S^{3} \backslash K$ ). The derivation of a presentation for $\mathcal{A}_{n}(M)$ follows the classical case (see page 122123 of [Hi2])but is complicated by basepoint concerns. The following overlaps with work of S. Harvey [Ha].

Proposition 6.1 The following sequence is exact.

$$
H_{1}\left(V ; \mathbb{K}_{n}\right) \otimes_{\mathbb{K}_{n}} \mathbb{K}_{n}\left[t^{ \pm 1}\right] \stackrel{d}{\rightarrow} H_{1}\left(Y ; \mathbb{K}_{n}\right) \otimes_{\mathbb{K}_{n}} \mathbb{K}_{n}\left[t^{ \pm 1}\right] \longrightarrow \mathcal{A}_{n}(M) \longrightarrow 0
$$

where $d(\alpha \otimes 1)=\left(i_{+}\right)_{*} \alpha \otimes t-\left(i_{-}\right)_{*} \alpha \otimes 1$.

Proof (see [Ha for a more detailed proof) For simplicity let $\Gamma_{n}$ stand for $G / G_{\mathbb{Q}}^{(n+1)}$ so there is an exact sequence $1 \longrightarrow \widetilde{G} \longrightarrow \Gamma_{n} \stackrel{\pi}{\rightarrow} \mathbb{Z} \longrightarrow 1$ where $\pi(u)=1$ and $\mathbb{K}_{n}$ is the quotient (skew) field of $\mathbb{Z} \widetilde{G}$. Let $U=V \times[-1,1]$ and consider a Mayer-Vietoris sequence for homology with $\mathbb{Z} \Gamma_{n}$ coefficients using the decomposition $M=Y \cup U$. Or, more naively, consider an ordinary MayerVietoris sequence for the integral homology of $M_{\Gamma_{n}}$, the $\Gamma_{n}$ cover, using the decomposition $M_{\Gamma_{n}}=p^{-1}(Y) \cup p^{-1}(U)=Y_{\Gamma_{n}} \cup U_{\Gamma_{n}}$ and note that all the maps are $\mathbb{Z} \Gamma_{n}$-module homomorphisms. After the usual simplification one arrives at the exact sequence:

$$
\longrightarrow H_{1}\left(V ; \mathbb{Z} \Gamma_{n}\right) \stackrel{d}{\rightarrow} H_{1}\left(Y ; \mathbb{Z} \Gamma_{n}\right) \stackrel{j_{*}}{\longrightarrow} \mathcal{A}_{n}^{\mathbb{Z}}(M) \stackrel{\partial_{*}}{\rightarrow} H_{0}\left(V ; \mathbb{Z} \Gamma_{n}\right) .
$$

Localizing yields a similar sequence with $\mathbb{K}_{n}\left[t^{ \pm 1}\right]$ coefficients where $\mathcal{A}_{n}(M)$ replaces $\mathcal{A}_{n}^{\mathbb{Z}}(M)$. Since $\pi_{1}(V)$ and $\pi_{1}(Y)$ are contained in $\widetilde{G}$, one can consider $H_{*}\left(V ; \mathbb{K}_{n}\right)$ and $H_{*}\left(Y ; \mathbb{K}_{n}\right)$, which are free $\mathbb{K}_{n}$-modules. Moreover $\mathbb{K}_{n}\left[t^{ \pm 1}\right]$ is free and hence flat as a left $\mathbb{K}_{n}$ module. Thus $H_{*}\left(V ; \mathbb{K}_{n}\left[t^{ \pm 1}\right]\right) \cong H_{*}\left(V ; \mathbb{K}_{n}\right) \otimes_{\mathbb{K}_{n}}$ $\mathbb{K}_{n}\left[t^{ \pm 1}\right]$ and $H_{*}\left(Y ; \mathbb{K}_{n}\left[t^{ \pm 1}\right]\right) \cong H_{*}\left(Y ; \mathbb{K}_{n}\right) \otimes_{\mathbb{K}_{n}} \mathbb{K}_{n}\left[t^{ \pm 1}\right]$, showing that these homology groups are finitely-generated free $\mathbb{K}_{n}\left[t^{ \pm 1}\right]$ modules. Since $\mathcal{A}_{n}(M)$ is a torsion module by Proposition 4.2 and $H_{0}\left(V ; \mathbb{K}_{n}\left[t^{ \pm 1}\right]\right)$ is free, $\partial_{*}$ is the zero map. This concludes our sketch of the proof of the proposition. 
Corollary 6.2 If the (classical) Alexander polynomial of $M$ is not 1 then $\mathcal{A}_{n}(M), n>0$, has a square presentation matrix of size $r=\max \{0,-\chi(V)\}$ each entry of which is a Laurent polynomial of degree at most 1. Specifically, we have the presentation

$$
\left(\mathbb{K}_{n}\left[t^{ \pm 1}\right]\right)^{r} \stackrel{\partial}{\rightarrow}\left(\mathbb{K}_{n}\left[t^{ \pm 1}\right]\right)^{r} \longrightarrow \mathcal{A}_{n}(M) \longrightarrow 0
$$

where $\partial$ arises from the above proposition. If $n=0$ then the same holds with $r$ replaced by $\beta_{1}(V)$.

Proof The Corollary will follow immediately from the Proposition if we establish that $H_{1}\left(V ; \mathbb{K}_{n}\right) \cong H_{1}\left(Y ; \mathbb{K}_{n}\right) \cong \mathbb{K}_{n}^{r}$. Note that both $V$ and $Y$ have the homotopy type of finite connected 2-complexes. Consider the coefficient systems $\psi: \pi_{1}(V) \longrightarrow \widetilde{G}$ and $\psi^{\prime}: \pi_{1}(Y) \longrightarrow \widetilde{G}$ obtained by restriction of $\pi_{1}(M) \longrightarrow \Gamma_{n}$. Letting $b_{i}$ stand for the rank of $H_{i}(; ; \widetilde{Z})$ or equivalently the rank of $H_{i}\left(\ldots ; \mathbb{K}_{n}\right)$, we have that $\chi(V)=b_{0}(V)-b_{1}(V)+b_{2}(V)$ as in Fact 3 .

Suppose that $\psi$ is non-trivial. Then $b_{0}(V)=0$ by Proposition 3.7. Since $\widetilde{G}$ is PTFA, it is torsion free and hence the image of $\psi$ is infinite. It follows that the $\widetilde{G}$-cover of $V$ is a non-compact 2 -manifold and thus $b_{2}(V)=0$. Therefore $b_{1}(V)=r$ as desired. It also follows that $\psi^{\prime}$ is non-trivial and so $b_{0}(Y)=0$. Since $\chi(M)=0$ it follows that $\chi(Y)=\chi(V)$. Thus $b_{2}(Y)-b_{1}(Y)=\chi(Y)=$ $\chi(V)=-b_{1}(V)$ so $b_{1}(Y)=b_{1}(V)+b_{2}(Y)$. By Proposition $6.1 \mathcal{A}_{n}$ has a presentation of deficiency $b_{1}(Y)-b_{1}(V)$. If $b_{2}(Y)>0$ then $\mathcal{A}_{n}(M)$ has a presentation of positive deficiency, contradicting the fact that it is a $\mathbb{K}_{n}\left[t^{ \pm 1}\right]-$ torsion module. Therefore $b_{2}(Y)=0$ and $b_{1}(Y)=b_{1}(V)=r$ as required. This completes the case that $\psi$ is non-trivial, after noting that if $n=0$ then $\psi$ is certainly trivial since $\widetilde{G}=1$.

Now suppose $\psi$ is trivial. If $n=0$ then this is the case of the classical (rational) Alexander module and the result is well-known. If $n \geq 1$ then the triviality of $\psi$ implies that $\pi_{1}(V) \subseteq G_{\mathbb{Q}}^{(2)}$. Consider a map $f: M \longrightarrow S^{1}$ such that $V$ is the inverse image of a regular value. Then $G_{\mathbb{Q}}^{(1)}=\operatorname{ker} f_{*}$ and it follows that $G_{\mathbb{Q}}^{(1)}$ is the normal subgroup generated by $\pi_{1}(Y)$ and so, for any $\gamma \in \pi_{1}(Y)$, there exists a non-zero integer $m$ such that $m \gamma$ bounds an orientable surface $S$. Hence $G_{\mathbb{Q}}^{(1)} / G_{\mathbb{Q}}^{(2)}$ is generated by $\pi_{1}(V)$ and thus is zero. It follows that $\mathcal{A}_{0}(M)=0$ and that classical Alexander polynomial is 1 . Since this case was excluded by hypothesis, the proof is complete.

Example 6.3 Suppose $K$ is a fibered knot of genus $g$ with fiber surface $V$ and $\pi_{1}$-monodromy $f$. If $n>0$ and $F$ is the free group of rank $2 g-1$ 
then $H_{1}\left(V ; \mathbb{K}_{n}\right) \cong H_{1}\left(Y ; \mathbb{K}_{n}\right) \cong H_{1}\left(F ; \mathbb{K}_{n}\right) \cong \mathbb{K}_{n}^{2 g-1}$ by Lemma 3.9. By the above results, $\mathcal{A}_{n}$ has a $(2 g-1)$ by $(2 g-1)$ presentation matrix given by It $-f_{n}$ where $f_{n}$ is an automorphism of the vector space $\mathbb{K}_{n}^{2 g-1}$ derived from the induced action of $f$ on $F / F^{(n+1)}$.

\section{The $\delta_{n}$ give lower bounds for knot genus}

The previous section can now be used to show that the degrees of the higher order Alexander polynomials give lower bounds for genus $(K)$. In the last part of this section we show that there are knots such that $\delta_{0}<\delta_{n}+1$ so that these invariants yield sharper estimates of knot genus than that given by the Alexander polynomial, $\operatorname{deg}\left(\Delta_{0}\right) \leq 2$ genus $(K)$. S. Harvey has established analagous results for any 3 -manifold, finding lower-bounds for the Thurston norm [Ha].

Theorem 7.1 If $K$ is a (null-homologous) non-trivial knot in a rational homology sphere and $\delta_{n}$ is the degree of the $n^{\text {th }}$ order Alexander polynomial then $\delta_{0} \leq 2 \operatorname{genus}(K)$ and $\delta_{n}+1 \leq 2 \operatorname{genus}(K)$ if $n>0$.

Proof. We may assume $n>0$ since the result for $n=0$ is well known. If the classical Alexander polynomial is 1 then $\delta_{0}=\delta_{n}=0$ and the theorem holds. Otherwise suppose $V$ is a Seifert surface of minimal genus. By Corollary 6.2 $\mathcal{A}_{n}(K)$ has a square presentation matrix of size 2 genus $(K)-1$. Since $\delta_{n}$ is defined as $\operatorname{rank}_{\mathbb{K}_{n}} \mathcal{A}_{n}$, it remains only to show that the latter is at most 2 genus $(K)-1$. This is accomplished by the following lemma of Harvey.

Lemma 7.2 Ha Suppose $\mathcal{A}$ is a torsion module over a skew Laurent polynomial ring $\mathbb{K}\left[t^{ \pm 1}\right]$ where $\mathbb{K}$ is a division ring. If $\mathcal{A}$ is presented by an $m \times m$ matrix $\theta$ each of whose entries is of the form $t a+b$ with $a, b \in \mathbb{K}$, then the rank of $\mathcal{A}$ as a $\mathbb{K}$-module is at most $m$.

Theorem 7.3 For any knot $K$ whose (classical) Alexander polynomial is not 1 and any positive integer $k$, there exists a knot $K_{*}$ such that

a) $\mathcal{A}_{n}\left(K_{*}\right) \cong \mathcal{A}_{n}(K)$ for all $n<k$.

b) $\delta_{n}\left(K_{*}\right)=\delta_{n}(K)$ for all $n<k$.

c) $\delta_{k}\left(K_{*}\right)>\delta_{k}(K)$.

d) $K_{*}$ can be taken to be hyperbolic or to be concordant to $K$. 
Corollary 7.4 Under the hypotheses of the theorem above, there exists a hyperbolic knot $K_{*}$, with the same classical Alexander module as $K$, for which $\delta_{0}\left(K_{*}\right)<\delta_{1}\left(K_{*}\right)<\cdots<\delta_{k}\left(K_{*}\right)$.

Proof of Theorem $\mathbf{7 . 3}$ Let $P=\pi_{1}\left(S^{3} \backslash K\right)$ and let $\alpha$ be an element of $P^{(k)}$ which does not lie in $P^{(k+1)}$. By Corollary 4.8 such $\alpha$ are plentiful. We now describe how to construct a knot $K_{*}=K(\alpha, k)$ which differs from $K$ by a single "ribbon move," i.e. $K_{*}$ is obtained by adjoining a trivial circle $J$ to $K$ and then fusing $K$ to this circle by a band as shown in Figure 2. Thus $K_{*}$ is concordant to $K$. From a group theory perspective, what is going on is simple. It is possible to add one generator and one relation that precisely kills that generator if one "looks" modulo $n^{\text {th }}$ order commutators, but does not kill that generator if one "looks" modulo $(n+1)^{\text {st }}$ order commutators. Details follow.
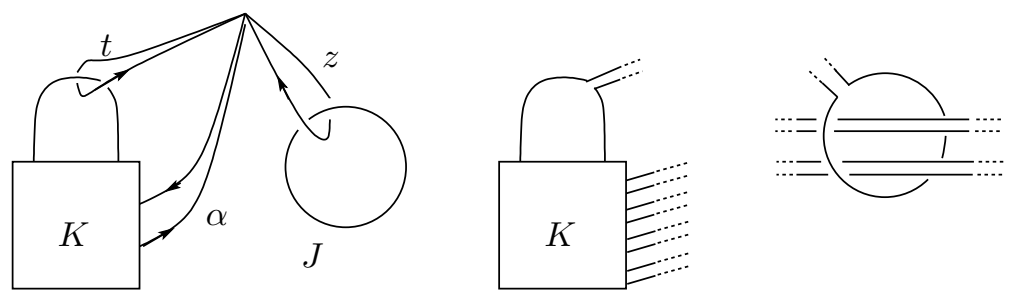

Figure $2: K_{*}$ is obtained from $K$ by a ribbon move

Choose meridians $t$ and $z$ as shown. Choose an embedded band which follows an arc in the homotopy class of the word $\eta=t\left[\alpha^{-1}, t^{-1} z\right] t^{-1}$. There are many such bands. For simplicity choose one which pierces the disk bounded by $J$ precisely twice corresponding to the occurrences of $z$ and $z^{-1}$ in $\eta$. Let $G=\pi_{1}\left(S^{3}-K_{*}\right)$ and let $\gamma$ denote a small circle which links the band. A Seifert Van-Kampen argument yields that the group $E \equiv G /\langle\gamma\rangle$ has a presentation obtained from a presentation of $P$ by adding a single generator $z$ (corresponding to the meridian of the trivial component) and a single relation $z=\eta t \eta^{-1}$. We symbolize this by $E=\left\langle P, z \mid z=\eta t \eta^{-1}\right\rangle$. First we analyze the relationship between $P$ and $E$.

Lemma 7.5 Given $P, \alpha, k, t, z, E$ as above:

a) $P / P^{(n)} \cong E / E^{(n)}$ for all $n \leq k+1$ implying that for all $n<k, \mathcal{A}_{n}^{\mathbb{Z}}(P) \cong$ $\mathcal{A}_{n}^{\mathbb{Z}}(E)$ and $\delta_{n}(P)=\delta_{n}(E)$;

b) $\delta_{k}(E)=\delta_{k}(P)+1$. 
Proof of Lemma 7.5 Let $w=t^{-1} z$ so $E=\left\langle P, w \mid w=\left[t^{-1}, \eta\right]\right\rangle$ and $\eta=t\left[\alpha^{-1}, w\right] t^{-1}$. Since $\alpha \in P^{(k)}, \eta \in E^{(k)}$ and hence $w \in E^{(k)}$. But then $\eta \in E^{(k+1)}$ so $w \in E^{(k+1)}$. Part a) of the Lemma follows immediately: the epimorphism $E \longrightarrow P$ obtained by killing $w$ induces an isomorphism $E / E^{(k+1)} \longrightarrow P / P^{(k+1)}$, and hence $\mathcal{A}_{n}^{\mathbb{Z}}(P) \cong \mathcal{A}_{n}^{\mathbb{Z}}(E)$ for $n<k$ by Definition 2.8. Here we use the fact that both $E$ and $P$ are $\mathcal{E}$-groups in the sense of R. Strebel (being fundamental groups of 2-complexes with $H_{2}=0$ and $H_{1}$ torsion-free). Consequently any term of their derived series is also an $\mathcal{E}$-group and it follows that their derived series is identical to their rational derived series Str].

Now we consider the subgroup $E^{(k+1)}$ of $E$. To justify the following grouptheoretic statements, consider a 2 -complex $X$ whose fundamental group is $P$ and define a 2-complex $Y$ by adjoining a 1-cell and a 2 -cell so that $\pi_{1}(Y) \cong E$ corresponding to the presentation $\left\langle P, w \mid w=\left[t^{-1}, \eta\right]\right\rangle$. The subgroup $E^{(k+1)}$ is thus obtained by taking the infinite cyclic cover $Y_{\infty}$ of $Y$ (so $\pi_{1}\left(Y_{\infty}\right)=E^{(1)}$ ) followed by taking the $E^{(1)} / E^{(k+1)}-\operatorname{cover} \widetilde{Y}$ of $Y_{\infty}\left(\right.$ so $\left.\pi_{1}(\tilde{Y})=E^{(k+1)}\right)$. Since the inclusion map $X \longrightarrow Y$ induces an isomorphism $P / P^{(k+1)} \longrightarrow E / E^{(k+1)}$, the induced cover of the subspace $X \subset Y$ is the cover $\widetilde{X}$ of $X$ with $\pi_{1}(X) \cong$ $P^{(k+1)}$. Therefore a cell structure for $\widetilde{Y}$ relative to $\widetilde{X}$ contains only the lifts of the 1-cell $w$ and the 2-cell corresponding to the single relation. This allows for an elementary analysis of $E^{(k+1)}$ as follows. By analyzing $X_{\infty}$ and $Y_{\infty}$ we see that

$$
E^{(1)}=\left\langle P^{(1)}, w_{i} i \in \mathbb{Z} \mid w_{i}=t^{-i}\left[t^{-1}, \eta\right] t^{i}\right\rangle
$$

where $w_{i}$ stands for $t^{-i} w t^{i}$ as an element of $\pi_{1}(Y)$. If we rewrite the relation using $\beta^{-1}=t^{-i} \alpha^{-1} t^{i}$ and $r^{-1}=t^{-i+1} \alpha^{-1} t^{i-1}$ we get

$$
E^{(1)}=\left\langle P^{(1)}, w_{i} \mid w_{i}=\beta^{-1} w_{i} \beta w_{i}^{-1} w_{i-1} r^{-1} w_{i-1}^{-1} r\right\rangle .
$$

This is a convenient form because what we want to do now is "forget the $t$ action" because $\delta_{k}$ is defined as the rank of the abelianization of $E^{(k+1)}$ as a module over $\mathbb{Z}\left[E^{(1)} / E^{(k+1)}\right]$ (or equivalently over its quotient field $\mathbb{K}_{k}$ ). Therefore we now think of $Y_{\infty}$ as being obtained from $X_{\infty}$ by adding an infinite number of 1 -cells $w_{i}$ and a correspondingly infinite number of 2 -cells. Thus $\tilde{Y}$ is obtained from $\widetilde{X}$ by adding 1 -cells $\left\{w_{i}^{s} \mid i \in \mathbb{Z}, s \in E^{(1)} / E^{(k+1)}\right\}$, where $w_{i}^{s}$ descends to $s^{-1} t^{-i} w t^{i} s$ in $E$, and 2-cells corresponding to the relations $\left\{w_{i}^{s}=\right.$ $\left.w_{i}^{\beta s}\left(w_{i}^{s}\right)^{-1} w_{i-1}^{s}\left(w_{i-1}^{r s}\right)^{-1} \mid i \in \mathbb{Z}, s \in E^{(1)} / E^{(k+1)}\right\}$ where, for example, $w_{i}^{\beta s}$ is the image of a fixed 1-cell $w_{i}$ under the deck translation $\beta s \in E^{(1)} / E^{(k+1)}$ and descends to $s^{-1} \beta^{-1} t^{-i} w t^{i} \beta s$ in $E$. The abelianization, $E^{(k+1)} / E^{(k+2)}$, as a right $\mathbb{Z}\left[E^{(1)} / E^{(k+1)}\right] \cong \mathbb{Z}\left[P^{(1)} / P^{(k+1)}\right]$ module is obtained from $P^{(k+1)} / P^{(k+2)}$ by adjoining a generator $w_{i}$ and a relation for each $i \in \mathbb{Z}$. Upon rewriting the 
relations above as $w_{i}(2 s-\beta s)_{*}=w_{i-1}(s-r s)_{*}$ where $(2 s-\beta s)_{*}$ denotes the (right) action of $2 s-\beta s \in \mathbb{Z}\left[P^{(1)} / P^{(k+1)}\right]$, and then again as $w_{i}(2-\beta)_{*} s_{*}=$ $w_{i-1}(1-r)_{*} s_{*}$ we see that the relations are generated as a module by $\left\{w_{i}(2-\right.$ $\left.\beta)_{*}=w_{i-1}(1-r)_{*} \mid i \in \mathbb{Z}\right\}$. Note that neither $2-\beta$ nor $1-r$ is zero since their augmentations are non-zero. Hence in $\mathbb{K}_{k}$ these elements are invertible and each $w_{i}, i \neq 0$ can be equated uniquely to a multiple of $w_{0}$. Thus $E^{(k+1)} / E^{(k+2)} \cong$ $P^{(k+1)} / P^{(k+2)} \oplus \mathbb{K}_{k}$ as a $\mathbb{K}_{k}$-module. It follows immediately that $\delta_{k}(E)=$ $\delta_{k}(P)+1$. This concludes the proof of Lemma 7.5

Returning to the proof of the theorem, it will suffice to show $\gamma \in G^{(k+1)}$ since if so then the epimorphism $G \longrightarrow E$ induces an isomorphism $G / G^{(n)} \cong E / E^{(n)}$ for all $n \leq k+1$ and hence an isomorphism $\mathcal{A}_{n}^{\mathbb{Z}}(G) \longrightarrow \mathcal{A}_{n}^{\mathbb{Z}}(E)$ for $n<k$. Moreover the epimorphism $G^{(k+1)} \longrightarrow E^{(k+1)}$ induces an epimorphism $\mathcal{A}_{k}^{\mathbb{Z}}(G) \longrightarrow$ $\mathcal{A}_{k}^{\mathbb{Z}}(E)$ of $G / G^{(k+1)}\left(\cong E / E^{(k+1)}\right)$ modules. Thus $\delta_{k}\left(K_{*}\right)=\delta_{k}(G) \geq \delta_{k}(E)$. By Lemma 7 7.5 the map $P \longrightarrow E$ induces isomorphisms $\mathcal{A}_{n}^{\mathbb{Z}}(P) \longrightarrow \mathcal{A}_{n}^{\mathbb{Z}}(E)$ for $n<k$ and $\delta_{k}(E)=\delta_{k}(P)+1=\delta_{k}(K)+1$. Combining these results will finish the proof.

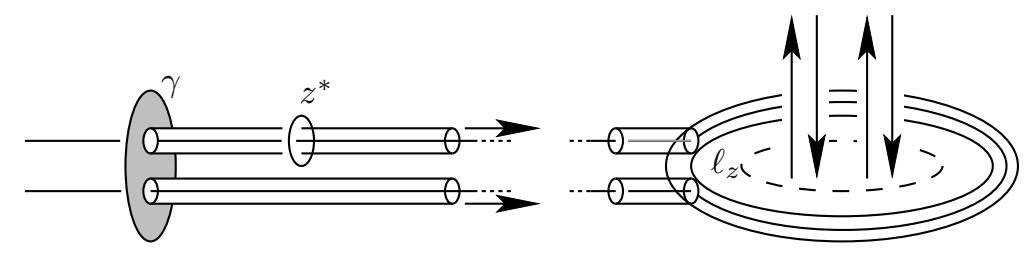

Figure 3: $\gamma=\left[z^{*}, \ell_{z}\right]$

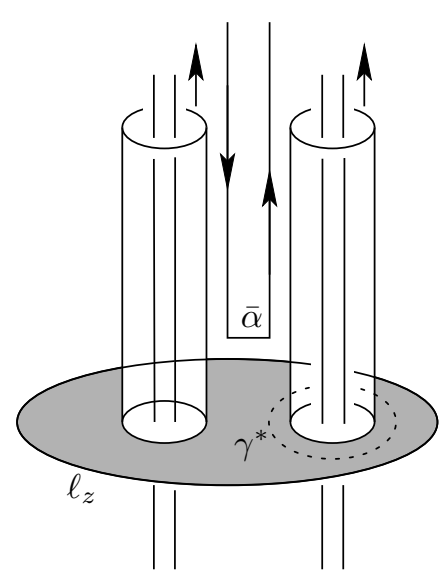

Figure 4: $\ell_{z}=\left[\gamma^{*}, \bar{\alpha}\right]$ 
To see that $\gamma \in G^{(k+1)}$, first note that $\gamma$ bounds an embedded disk which is punctured twice by the knot. By tubing along the knot in the direction of $J$, one sees that $\gamma$ bounds an embedded (punctured) torus in $S^{3} \backslash K_{*}$ as in Figure 3. This illustrates the group-theoretic fact that $\gamma=\left[z^{*}, \ell_{z}\right]$ where $\ell_{z}$ is a longitude of $J$ and $z^{*}$ is a conjugate of $z$. It suffices to show $\ell_{z} \in G^{(k+1)}$. But, since $\eta=t \alpha^{-1} t^{-1} z \alpha z^{-1}$ contains 2 occurrences of $z$ (with opposite sign) and we chose our band to pass precisely 2 times through $J, \ell_{z}$ bounds a twice punctured disk and hence a punctured torus as in Figure 4. This illustrates that $\ell_{z}=\left[\gamma^{*}, \bar{\alpha}\right]$ where $\gamma^{*}$ is a conjugate of $\gamma$ since it is another meridian of the band, and $\bar{\alpha}$ is the word $\alpha$ separating the occurrences of $z$ and $z^{-1}$ in the word $\eta$. Clearly $\gamma \in G^{(1)}$. Suppose $\gamma$, and hence $\gamma^{*}$, lies in $G^{(j)}$ for some $1 \leq j \leq k$. Thus $G / G^{(j)} \cong E / E^{(j)}$. Let $\alpha^{\prime}$ denote the image of $\bar{\alpha}$ under the map $G \rightarrow E$. Then $\alpha^{\prime}$ is the image of $\alpha$ under the map $P \rightarrow E$ since all the elements $\alpha, \alpha^{\prime}$ and $\bar{\alpha}$ are represented by the "same" path. Since $\alpha \in P^{(k)}$ (by hypothesis), $\alpha^{\prime} \in E^{(k)}$ and hence $\bar{\alpha} \in G^{(j)}$. But then $\ell_{z} \in G^{(j+1)}$ and hence $\gamma \in G^{(j+1)}$. Continuing in this way shows that $\gamma \in G^{(k+1)}$ and concludes the proof of Theorem 7.3 .

Proof of Corollary [7.4 By induction and Theorem 7.3 there exists a knot $K_{k-1}$ with the same classical Alexander module as $K$ and $\delta_{0}\left(K_{k-1}\right)<\ldots<$ $\delta_{k-1}\left(K_{k-1}\right)$ Apply Theorem 7.3 to $K_{k-1}$ produce a new knot $K_{*}$. One easily checks that $K_{*}$ satisfies the required properties by Theorem [7.3. Theorem 5.4 and Corollary 2.10.

\section{Genetic infection: A technique for constructing knots}

We discuss a satellite construction, which we call genetic modification or infection, by which a given knot $K$ is subtly modified, or infected using an auxiliary knot or link $J$ (see also of [COT1, Section 6] COT2 [CT]). If, by analogy, we think of the group $G$ of $K$ as its strand of DNA, then, by Corollary 4.8 this "strand" is infinitely long as measured by the derived series. Thus, as we shall see, it is possible to locate a spot on the "strand" which corresponds to an element of $G^{(n)}-G^{(n+1)}$, excise a "small piece of DNA" and replace it with "DNA associated to the knot $J$ ", with the effect that $G / G^{(n+1)}$ is not altered but $G / G^{(n+2)}$ is changed in a predictable fashion. The infection is subtle enough so that it is not detected by the localized modules $\mathcal{A}_{n}$ (hence not by $\left.\delta_{n}\right)$. The effect on the (integral) modules $\mathcal{A}_{n}^{\mathbb{Z}}$ can be measured numerically by the higher-order signatures of Section [1]. 
Suppose $K$ and $J$ are fixed knots and $\eta$ is an embedded oriented circle in $S^{3} \backslash K$ which is itself unknotted in $S^{3}$. Note that any class $[\eta] \in G$ has a (non-unique) representative $\eta$ which is unknotted in $S^{3}$. Then $(K, \eta)$ is isotopic to part a of Figure 5 below, where some undetermined number $m$ of strands of $K$ pierce the disk bounded by $\eta$. Let $K_{0}=K(\eta, J)$ be the knot obtained by replacing the $m$ trivial strands of $K$ by $m$ strands "tied into the knot $J$ ". More precisely, replace them with $m$ untwisted parallels of a knotted arc with oriented knot type $J$ as in Figure 5 . We call $K_{0}$ the result of infecting $K$ by $J$ along $\eta$.

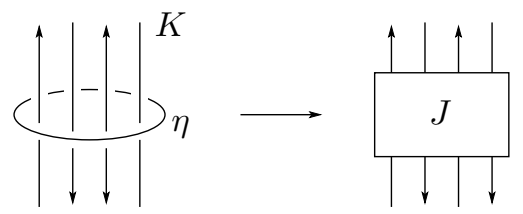

Figure 5: Infecting $K$ by $J$ along $\eta$

The more general procedure of replacing the $m$ strands by a more complicated string link will be discussed briefly in Section 10. Note that this is just a satellite construction and as such is not new. The emphasis here is on choosing the loop or loops $\eta$ to be very subtle with respect to some measure. Note that this construction is, in a sense, orthogonal to techniques used by Casson-Gordon, Litherland, Gilmer, T.Stanford, and K.Habiro wherein the loop $\eta$ is arbitrary but the analogue of the infection parameter $J$ is increasingly subtle (for example, in Stanford's case, $J$ must lie in the $n^{\text {th }}$ term of the lower central series of the pure braid group; and, in the claspers that Habiro associated to Vassiliev theory, the analogue of $\eta$ is a meridian of $K[\mathrm{Hb}]$ ). However, infection can certainly be viewed as the result of modifying $K$ by a certain clasper (depending on $J$ ) all of whose leaves are parallels of $\eta$ (see [CT] GL [GR]). Moreover all of these procedures are special cases of the classical technique, used by J. Levine and others, of modifying a knot by Dehn surgeries that leave the ambient manifold unchanged.

We now give an alternate description of genetic infection that is better suited to analysis by Mayer-Vietoris and Seifert-Van Kampen techniques. Beginning with the exterior of $K, E(K)$, delete the interior of a tubular neighborhood $N$ of $\eta$ and replace it with the exterior of $J, E(J)$, identifying the meridian $\mu_{\eta}$ of $\eta$ with the longitude $\ell_{J}$ of $J$, and the longitude $\ell_{\eta}$ of $\eta$ with the meridian $\mu_{J}$ of $J$. It is well-known and is a good exercise for the reader to show that the resulting space is $E\left(K_{0}\right)$ as described above. Note that this replaces the exterior of a unknot with the exterior of the knot $J$ in a fashion that preserves homology. Since there is a degree one map (rel boundary) from $E(J)$ to $E$ (unknot), there 
is a degree one map (rel boundary) $f$ from $E\left(K_{0}\right)$ to $E(K)$ which is the identity outside $E(J)$.

Theorem 8.1 If $\eta \in G^{(n)}$ then the map $f$ (above) induces an isomorphism $f: \pi_{1}\left(E\left(K_{0}\right)\right) / \pi_{1}\left(E\left(K_{0}\right)\right)^{(n+1)} \rightarrow \pi_{1}(E(K)) / \pi_{1}(E(K))^{(n+1)}$ and hence induces isomorphisms between the $i^{\text {th }}$ (integral and localized) modules of $K_{0}$ and $K$ for $0 \leq i<n$.

Proof Let $E(\eta)$ denote $E(K)$ with the interior of an open tubular neighborhood of $\eta$ deleted. Then, by the Seifert-VanKampen theorem, $G=\pi_{1}(E(K)) \cong$ $\left\langle\pi_{1}(E(\eta)), t \mid \mu_{\eta}=1, \ell_{\eta}=t\right\rangle$. Similarly, $G_{0}=\pi_{1}\left(E\left(K_{0}\right)\right) \cong\left\langle\pi_{1}(E(\eta)), \pi_{1}(E(J))\right.$ $\left|\ell_{\eta}=\mu_{J}, \mu_{\eta}=\ell_{J}\right\rangle$ where this denotes the obvious "free product with amalgamation". The map $f$ induces the identity on $\pi_{1}(E(\eta))$ and is the Hurewicz map on $\pi_{1}(E(J)) \rightarrow \mathbf{Z}=\langle t\rangle$ which sends $\ell_{J} \rightarrow 1$ and $\mu_{J} \rightarrow t$. Hence the kernel of $f: G_{0} \rightarrow G$ is precisely the normal closure in $G_{0}$ of $[P, P]$ where $P=\pi_{1}(E(J))$. Thus it suffices to show that $P \subset G_{0}^{(n)}$. Since $P$ is normally generated by $\mu_{J}$, it suffices to show by induction that $\mu_{J} \in G_{0}^{(n)}$. This is clearly true for $n=0$. Suppose $\mu_{J} \in G_{0}^{(k)} k<n$. Then $P \subset G_{0}^{(k)}$ so $\mu_{\eta}=\ell_{J} \subset[P, P] \subset G_{0}^{(k+1)}$. By hypothesis $\eta \in G^{(n)}$. Therefore $\eta$ bounds in $E(K)$, a map of a symmetric $n$-stage grope [CTe. Thus $\ell_{\eta}$ bounds such a grope in $E(K)$ and we may assume that the grope stages meet $\eta$ transversely. Hence $\ell_{\eta}$ bounds a punctured $n$-stage grope in $E(\eta)$ and the boundaries of these punctures are copies of $\mu_{\eta}$. Therefore, in $G_{0}, \ell_{\eta}=\prod_{i=1}^{m} \xi_{i} \mu_{\eta}^{n_{i}} \xi_{i}^{-1} \prod_{j=1}^{r}\left[a_{j}, b_{j}\right]$ where each $a_{j}$ and $b_{j}$ bound maps of punctured $(n-1)$-stage gropes in $E(\eta)$. We claim $\ell_{\eta} \in G_{0}^{(k+1)}$. It suffices to show the $a_{j}$ and $b_{j}$ lie in $G_{0}^{(k)}$. But each of these, modulo conjugates of $\mu_{\eta}^{ \pm 1}$, is given by a similar expression as $\ell_{\eta}$ above. Continuing in this fashion, we see that $\ell_{\eta} \in G_{0}^{(n)}$ modulo the punctures $\mu_{\eta} \in G_{0}^{(k+1)}$. Since $n \geq k+1$, $\ell_{\eta} \in G_{0}^{(k+1)}$ and hence $\mu_{J} \in G_{0}^{(k+1)}$, completing our induction.

Theorem 8.2 Let $K_{0}=K(\eta, J)$ be the result of genetic infection of $K$ by $J$ along $\eta \in G^{(n)}$ (as described above). Then the $n^{\text {th }}$ (integral) Alexander module of $K_{0}, \mathcal{A}_{n}^{\mathbb{Z}}\left(K_{0}\right)$, is isomorphic to $\mathcal{A}_{n}^{\mathbb{Z}}(K) \oplus\left(\mathcal{A}_{0}^{\mathbb{Z}}(J) \otimes_{\mathbb{Z}\left[t, t^{-1}\right]} \mathbb{Z}\left[G / G^{(n+1)}\right]\right)$ where $\mathbb{Z}\left[G / G^{(n+1)}\right]$ is a left $\mathbb{Z}\left[t, t^{-1}\right]$ module via the homomorphism $\langle t\rangle=\mathbb{Z} \rightarrow$ $G / G^{(n+1)}$ sending $t \rightarrow \eta$. Thus, if $n \geq 1, \mathcal{A}_{i}\left(K_{0}\right) \cong \mathcal{A}_{i}(K)$ for all $i \leq n$.

Proof Note that since $\eta \in G^{(n)}, \mathcal{A}_{n}^{\mathbb{Z}}\left(K_{0}\right)$ and $\mathcal{A}_{n}^{\mathbb{Z}}(K)$ are modules over isomorphic rings since $G / G^{(n+1)} \cong G_{0} /\left(G_{0}\right)^{(n+1)}$ by the previous theorem. Therefore we can take the point of view that the map $E\left(K_{0}\right) \rightarrow E(K)$ induces on both spaces a local coefficient system with $G / G^{(n+1)}$ coefficients. 
Lemma 8.3 The inclusion $i: \partial E(J) \rightarrow E(J)$ induces an isomorphism on $H_{0}\left(\_; \mathbb{Z}\left[G / G^{(n+1)}\right]\right)$ and induces either the 0 map or an epimorphism on $H_{1}\left(\_; \mathbb{Z}\left[G / G^{(n+1)}\right]\right.$ according as $\eta \notin G^{(n+1)}$ or $\eta \in G^{(n+1)}$ respectively, whose kernel is generated by $\left\langle\ell_{J}\right\rangle$.

Proof of Lemma 8.3 The Lemma refers to the coefficient system on $E(J)$ induced by $E(J) \subseteq E\left(K_{0}\right) \rightarrow E(K)$. Note that the kernel of the map $\pi_{1}(E(J)) \rightarrow$ $G$ contains $\left[\pi_{1}(E(J)), \pi_{1}(E(J))\right]$ and thus its image in $G / G^{(n+1)}$ is cyclic, generated by the image of $\mu_{J}=\eta$. Since $G / G^{(n+1)}$ is torsion-free (see Example $2.4)$, this image is either zero or $\mathbb{Z}$ according as $\eta \in G^{(n+1)}$ or not. This also shows that the image of $\pi_{1}(\partial E(J))$ in $G / G^{(n+1)}$ is the same as the image of $\pi_{1}(E(J))$. The first claim of the Lemma now follows immediately from the proof of Proposition [3.7. Alternatively, since $H_{0}\left(\_; \mathbb{Z}\left[G / G^{(n+1)}\right]\right)$ is free on the path components of the induced cover, and since the cardinality of such is the index of the image of $\pi_{1}$ in $G / G^{(n+1)}, i$ induces an isomorphism on $H_{0}\left(\_; \mathbb{Z}\left[G / G^{(n+1)}\right]\right)$. If $\eta \in G^{(n+1)}$ then the induced local coefficient systems on $\partial E(J)$ and $E(J)$ are trivial, i.e. untwisted and thus $i$ induces an epimorphism on $H_{1}\left(\_; \mathbb{Z}\left[G / G^{(n+1)}\right]\right)$ whose kernel is $\left\langle\ell_{J}\right\rangle$ because it does so with ordinary $\mathbb{Z}$ coefficients. If $\eta \notin G^{(n+1)}$ then the induced cover of $\partial E(J)$ is a disjoint union of copies of the $\mathbb{Z}$-cover which "unwinds" $\mu_{J}$, i.e. the ordinary infinite cyclic cover. Thus $H_{1}$ of this cover is generated by a lift of $\ell_{J}$. But $\ell_{J}$ bounds a surface in $E(J)$ and this surface lifts to the induced cover since every loop on a Seifert surface lies in $\left[\pi_{1}(E(J)), \pi_{1}(E(J))\right]$. Therefore $i$ induces the zero map on $H_{1}$ in this case. This concludes the proof of the Lemma.

We return to the proof of Theorem 8.2 Consider the Mayer-Vietoris sequence with $\mathbb{Z}\left[G / G^{(n+1)}\right]$ coefficients for $E\left(K_{0}\right)$ viewed as $E(J) \cup E(\eta)$ with intersection $\partial E(J)$. By Lemma 8.3 this simplifies to

$$
H_{1}(\partial E(J)) \stackrel{\left(\psi_{1}, \psi_{2}\right)}{\longrightarrow} H_{1}(E(J)) \oplus H_{1}(E(\eta)) \longrightarrow H_{1}\left(E\left(K_{0}\right)\right) \longrightarrow 0 .
$$

Note first that $E(K)$ is obtained from $E(\eta)$ by adding a solid torus, i.e. a 2-cell and then a 3-cell, so that it is clear that $H_{1}(E(K))$ is the quotient of $H_{1}(E(\eta))$ by the submodule generated by $\mu_{\eta}$ (or $\left.\ell_{J}\right)$. If $\eta \notin G^{(n+1)}$ then $\psi_{1}$ is zero by Lemma 8.3] so $H_{1}\left(E\left(K_{0}\right)\right) \cong H_{1}(E(J)) \oplus\left(H_{1}(E(\eta)) /\left\langle\psi_{2}\right\rangle\right)$. But in the proof of Lemma 8.3 we saw that $H_{1}(\partial E(J))$ was generated by $\ell_{J}$ and so the image of $\psi_{2}$ is generated by $\ell_{J}$. Hence $H_{1}\left(E\left(K_{0}\right)\right) \cong H_{1}(E(J)) \oplus\left(H_{1}(E(K))\right.$. This concludes the proof of the theorem in the case $\eta \notin G^{(n+1)}$ once we identify $H_{1}(E(J))$ as $\mathcal{A}_{0}^{\mathbb{Z}}(J) \otimes_{\mathbb{Z}\left[t, t^{-1}\right]} \mathbb{Z}\left[G / G^{(n+1)}\right]$. But since the map from $\pi_{1}(E(J))$ to its image in $G / G^{(n+1)}$ has already been observed to be the abelianization, this is clear. 
In case $\eta \in G^{(n+1)}, \psi_{1}$ is an epimorphism whose kernel is generated by $\ell_{J}$ and so $H_{1}\left(E\left(K_{0}\right)\right) \cong H_{1}(E(\eta)) /\left\langle\ell_{J}\right\rangle \cong H_{1}(E(K))$. On the other hand, in this case $\mathcal{A}_{0}^{\mathbb{Z}}(J) \otimes_{\mathbb{Z}\left[t, t^{-1}\right]} \mathbb{Z}\left[G / G^{(n+1)}\right]$ factors through the augmentation of $\mathcal{A}_{0}^{\mathbb{Z}}(J)$, which is zero since the classical Alexander polynomial of a knot augments to 1.

If $n \geq 1, \eta \in \widetilde{G}$. Let $\Delta(t)$ be the classical Alexander polynomial of $J$. Then $\Delta(\eta) \in \mathbb{Z} \widetilde{G}-\{0\}$. Recall that $\mathbb{Z} \widetilde{G}-\{0\}$ is a right divisor set of regular elements of $\mathbb{Z}\left[G / G^{(n+1)}\right]$ by $[\mathrm{P}$. p. 609$]$. Thus for any $r \in \mathbb{Z}\left[G / G^{(n+1)}\right]$, there exist $r_{1} \in \mathbb{Z}\left[G / G^{(n+1)}\right]$ and $t_{1} \in \mathbb{Z} \widetilde{G}-\{0\}$ such that $\Delta(\eta) r_{1}=r t_{1}[\mathrm{P}$ p. 427]. Hence any element $x \otimes r \in \mathcal{A}_{0}^{\mathbb{Z}}(J) \otimes \mathbb{Z}\left[G / G^{(n+1)}\right]$ is annihilated by $t_{1}$, showing that this is a $\mathbb{Z} \widetilde{G}$-torsion module. Hence $\mathcal{A}_{n}\left(K_{0}\right) \cong \mathcal{A}_{n}(K)$.

\section{Applications to detecting fibered and alternating knots and symplectic structures on 4-manifolds}

In this section we show that the higher-order Alexander modules of fibered knots and alternating knots have special properties. Therefore noncommutative knot theory gives algebraic invariants which can be used to tell when a knot is not fibered or not alternating, even in situations where the Alexander module yields inconclusive evidence. In the case of fibered knots, examples of this type were obtained independently by J.C. Cha using the twisted Alexander invariant Ch. Remarkably, for 4 -manifolds of the form $M_{K} \times S^{1}$ ( $M_{K}$ is the 0-surgery on $K$ ), our invariants also obstruct the existence of a symplectic structure (using work of P. Kronheimer $[\mathbf{K r}]$ ). We also establish that $\delta_{i}-\delta_{j}$ are not Vassiliev invariants of finite type.

Proposition 9.1 If $K$ is a non-trivial fibered or alternating knot then $\delta_{0}=$ $\delta_{1}+1=\cdots=\delta_{n}+1=2 \operatorname{genus}(K)$.

Proof It is well known that for a fibered or alternating knot, $\delta_{0}=2$ genus $(K)$. The result now follows immediately from Theorem 5.4

Corollary 9.2 For any non-trivial fibered or alternating knot $K$, and any positive integer $n$, there exists a hyperbolic knot $K_{*}$ such that

a) $\mathcal{A}_{k}\left(K_{*}\right) \cong \mathcal{A}_{k}(K)$ for all $k<n$

b) $\delta_{k}\left(K_{*}\right)=\delta_{k}(K)$ for all $k<n$

c) $\delta_{n}\left(K^{*}\right)>\delta_{n}(K)$ 
d) $K_{*}$ is neither fibered nor alternating.

Proof Apply Theorem 7.3 and Corollary 2.10 to produce $K_{*}$. Suppose $n \geq 2$. Since $K$ is fibered or alternating, $\delta_{n}(K)=\delta_{n-1}(K)$ by Proposition 9.1 It follows that $\delta_{n}\left(K_{*}\right)>\delta_{n-1}\left(K_{*}\right)$ so $K_{*}$ is not fibered. A similar argument works for $n=1$.

There are more subtle obstructions to fibering that cannot be detected by the localized modules, but can be detected by the integral modules.

Proposition 9.3 If $K$ is a fibered knot then the following equivalent conditions hold:

1) $\mathcal{A}_{n}^{\mathbb{Z}}(K) \longrightarrow \mathcal{A}_{n}(K)$ is injective

2) $\mathcal{A}_{n}^{\mathbb{Z}}(K)$ is torsion-free as a $\mathbb{Z} \widetilde{G}$-module (recall that $\widetilde{G}$ is $G^{(1)} / G^{(n+1)}$ ).

Proof Recall $\mathcal{A}_{n}^{\mathbb{Z}}(K)=G^{(n+1)} / G^{(n+2)}=F^{(n)} / F^{(n+1)}$ where $G^{(1)}=F$ is free since $K$ is a fibered knot. Since $\widetilde{G}=G^{(1)} / G^{(n+1)}=F / F^{(n)}, \mathcal{A}_{n}^{\mathbb{Z}}$ as a $\mathbb{Z} \widetilde{G}-$ module is merely $F^{(n)} / F^{(n+1)}$ as a $\mathbb{Z}\left[F / F^{(n)}\right]$-module (i.e. $H_{1}\left(F ; \mathbb{Z}\left[F / F^{(n)}\right]\right)$ ). Since $F$ is the fundamental group of a 1 -complex, this is a submodule of a free module and hence is torsion-free.

Theorem 9.4 For any non-trivial fibered knot $K$ and any positive integer $n$ there exists a family of hyperbolic knots $K_{*}=K_{*}(J, n)$, parametrized by an auxiliary knot $J$, such that

1) $G / G^{(n+1)} \cong G_{*} / G_{*}^{(n+1)}$ meaning that all knots in the family share (with $K)$ the same $\mathcal{A}_{i}^{\mathbb{Z}}$ for $0 \leq i \leq n-1$;

2) $\mathcal{A}_{n}(K) \cong \mathcal{A}_{n}\left(K_{*}\right)$

3) $\delta_{0}=\delta_{1}+1=\cdots=\delta_{n}+1$ for each $K_{*}$ and $K$

4) if $P_{*}$ is the commutator subgroup of $G_{*}$ then $P_{*} /\left(P_{*}\right)_{j} \cong F / F_{j}$ for each term of the lower central series ( $F$ is free of rank equal to 2 genus $(K)$ ).

5) If $J$ has non-trivial classical Alexander polynomial then $K_{*}$ is not fibered and hence is distinct from $K$.

6) If $J$ has non-trivial classical Alexander polynomial then $G / G^{(n+2)} \not$ $G_{*} / G_{*}^{(n+2)}$ and $\mathcal{A}_{n}^{\mathbb{Z}}\left(K_{*}\right) ¥ \mathcal{A}_{n}^{\mathbb{Z}}(K)$.

7) $K_{*}(J, n)$ and $K_{*}\left(J^{\prime}, n\right)$ are distinct if the integrals of the classical Levine signature functions of $J$ and $J^{\prime}$ are distinct. 
Proof Since $K$ is a non-trivial fibered knot, it does not have Alexander polynomial 1. By Corollary 4.8 (or more simply since $G^{(n)}$ is free if $n \geq 1$ ), for any $n$, we can choose a class $\eta \in G^{(n)}-G^{(n+1)}$ which can be represented by a loop in the complement of a fiber surface for $K$ and which is also unknotted in $S^{3}$. Construct $K_{0}=K(\eta, J)$ by genetic infection as in Section 8 It follows that $\operatorname{genus}\left(K_{0}\right)=\operatorname{genus}(K)$ so if we drop the claim of hyperbolicity we can retain this. By Corollary 2.10 there is a hyperbolic knot, $K_{*}$, whose fundamental group differs from that of $K_{0}$ by a perfect group. Thus $K_{0}$ and $K_{*}$ have isomorphic $\mathcal{A}_{i}^{\mathbb{Z}}$ for any $i$ and have isomorphic groups modulo any term of the derived series (see Proposition 2.9). Thus, by Theorem 8.1 part 1) of Theorem 9.4 follows. Part 2) follows from Theorem 8.2. Part 3) holds for $K$ by Proposition 9.1 and hence for $K_{*}$ by the second part of Theorem 8.2, Part 4) is true for any knot for which $\mathcal{A}_{0}^{\mathbb{Z}} \cong \mathbb{Z}^{2 \text { genus }(K)}$ since one can then define a homomorphism from the free group of rank 2 genus $(K)$ to the commutator subgroup which induces an isomorphism on $H_{1}$ and an epimorphism on $H_{2}$. Stallings' theorem $\mathrm{St}$ then guarantees an isomorphism modulo any term of the lower central series.

By Theorem $8.2, \mathcal{A}_{n}^{\mathbb{Z}}\left(K_{*}\right)$ is $\mathcal{A}_{n}^{\mathbb{Z}}(K)$ direct sum $\mathcal{A}_{0}^{\mathbb{Z}}(J) \otimes_{\mathbb{Z}\left[t, t^{-1}\right]} \mathbb{Z}\left[G / G^{(n+1)}\right]$. But $\mathcal{A}_{n}\left(K_{*}\right) \cong \mathcal{A}_{n}(K)$. By Proposition 9.3, if $K_{*}$ were fibered then this second direct summand would be zero. But even after tensoring with $\mathbb{Q}\left[G / G^{(n+1)}\right]$ this module is not zero because it is cyclic of order $\Delta(\eta)$ where $\Delta(t)$ is the classical Alexander polynomial of $J$. Thus the module is zero if and only if $\Delta(\eta)$ is a unit in $\mathbb{Q}\left[G / G^{(n+1)}\right]$. Since $G / G^{(n+1)}$ is PTFA it is right orderable by $[\mathrm{P}$, p. 587] hence has only trivial units by $[\mathrm{P}$, p. 588,590$]$. Since $\Delta(\eta)$ is an integral polynomial in $\eta$, this can only happen if $\Delta(t)$ has degree zero which was excluding by hypothesis. Thus Part 5 is established. Part 6 follows from the discussion above. The proof of Part 7) must be postponed to Theorem 11.1 of Section [1].

Some of these new obstructions to fibering can be used to show that certain 4-manifolds of the form $S^{1} \times M_{K}$ admit no symplectic structure. If $K$ is a fibered knot then $M_{K}$ also fibers over the circle and it is known that $S^{1} \times$ $M_{K}$ is then symplectic. C. Taubes conjectured the converse. The SeibergWitten invariants provide evidence for this conjecture. If $S^{1} \times M_{K}$ admits a symplectic structure then the Alexander polynomial of $K$ must be monic. This is precisely the fibering obstruction on the classical Alexander polynomial of $K$. Peter Kronheimer provided more evidence for the conjecture by proving that if $S^{1} \times M_{K}$ admits a symplectic structure then $\delta_{0}=2 \operatorname{genus}(K)[\mathrm{Kr} 2[\mathrm{Kr}$. As a consequence of his work, we see that the $\delta_{i}$ constitute algebraic invariants 
which can obstruct a symplectic structure on $S^{1} \times M_{K}$ even when the Seiberg Witten invariants give inconclusive information.

Theorem 9.5 Suppose $K$ is a non-trivial knot. If $S^{1} \times M_{K}$ admits a symplectic structure then the invariants $\delta_{i}(K)-\delta_{0}(K)+1$ are zero for all $i>0$.

Proof By Kronheimer's theorem, $\delta_{0}(K)=2$ genus $(K)$. The result then follows from Theorem 5.4 .

Corollary 9.6 If $K_{*}$ is any one of the examples of Corollary 9.2 then $S^{1} \times M_{K}$ admits no symplectic structure although the Alexander polynomial of $K$ is monic.

Now consider $\delta_{n}$ as a rational valued invariant on knot types.

Proposition 9.7 None of the invariants $\delta_{i}-\delta_{j}(i \neq j)$ or $\delta_{i}-2 \operatorname{genus}(K)$ is determined by any finite number of finite type (Vassilliev) invariants.

Proof Let $\phi$ be one of the mentioned invariants. Suppose $\phi$ were determined by the finite type invariants $v_{1}, \ldots, v_{m}$. We have shown in Theorem 7.3 that $\phi$ is not constant. But on fibered knots $\phi$ is constant, say $C$, by Proposition 9.1 . Again using Theorem 7.3 choose a knot $K_{*}$ such that $\phi\left(K_{*}\right)=C^{\prime} \neq C$. By a result of A. Stoimenow [Sti], there exists a fibered knot $K$ such that $v_{i}(K)=$ $v_{i}\left(K_{*}\right)$ for $1 \leq i \leq m$. Thus $\phi(K)=\phi\left(K_{*}\right)$ a contradiction.

\section{Bordism invariants generalizing the Arf invariant}

In this section we define higher-order bordism invariants for knots which (in a certain sense) generalize the Arf invariant. The reader is warned that these are not the same as the generalizations of the Arf invariant defined in Section 4 of COT1. The invariants about to be defined are almost certainly not concordance invariants. If $K$ is a knot, $G$ its group, let $M_{K}$ be the result of 0 -framed surgery on $K$ and $P=\pi_{1}\left(M_{K}\right)$. Recall that the Arf invariant of $K$ may be defined as the class in $\Omega_{3}^{\text {Spin }}\left(S^{1}\right) \cong \mathbb{Z}_{2}$ represented by $M_{K}$ with the map to $S^{1}$ induced by the abelianization homomorphism $P \longrightarrow P / P^{(1)} \cong G / G^{(1)} \cong \mathbb{Z}$. Equivalently one could consider spin bordism (rel boundary) over $S^{1}$ of 3 manifolds with a toral boundary component, in which case the Arf invariant of $K$ is zero if and only if $S^{3} \backslash K$ is bordant to the exterior of the unknot. Note that $S^{1}=K\left(P / P^{(1)}, 1\right)=K\left(G / G^{(1)}, 1\right)$.

More generally, 
Definition 10.1 The $n^{\text {th }}$ (reduced) bordism invariant of $K, \beta_{n}(K)$, is the class in $\Omega_{3}^{\operatorname{Spin}}\left(K\left(P / P^{(n+1)}, 1\right)\right) / \operatorname{Aut}\left(P / P^{(n+1)}\right)$ represented by $M_{K} \stackrel{f_{n}}{\longrightarrow}$ $K\left(P / P^{(n+1)}, 1\right)$ where $f_{n}$ is induced by the quotient map $f_{n}: P \longrightarrow P / P^{(n+1)}$.

Obviously then $\beta_{0}(K)$ is the Arf invariant of $K$, and equivalent knots (here we need an orientation-preserving homeomorphism) have identical bordism invariants. Note also that $\beta_{n}(-K)=-\beta_{n}(K)$, so that a \pm -amphichiral knot satisfies $2 \beta_{n}=0$. We also have the following purer but uglier version. The purest (and ugliest) version would fix the peripheral structure in $G / G^{(n+1)}$ and effectively only consider pairs of knots with isomorphic $G / G^{(n+1)}$ preserving peripheral structure.

Definition 10.2 The $n^{\text {th }}$ unreduced bordism invariant of $K, \widetilde{\beta}_{n}(K)$, is the equivalence class of $\left(S^{3} \backslash K, \partial\left(S^{3} \backslash K\right), f_{n}: S^{3} \backslash K \longrightarrow K\left(G / G^{(n+1)}, 1\right)\right)$ in the set of spin bordism classes rel boundary of spin 3 -manifolds and maps to $K\left(G / G^{(n+1)}, 1\right)$ modulo the action of $\operatorname{Aut}\left(G / G^{(n+1)}, 1\right)$.

Conjecture For each $n \geq 0$ there exist knots $K, K_{*}$ such that $\mathcal{A}_{i}(K) \cong$ $\mathcal{A}_{i}\left(K_{*}\right), 0 \leq i<n$ but $\beta_{n}(K) \neq \beta_{n}\left(K_{*}\right)$.

We give a construction which should produce $K_{*}$ for any $n$ but are only able to verify this in the first case $n=0$.

Theorem 10.3 There exist knots $K, K_{*}$ with identical classical Alexander module and Blanchfield form but which are distinguished by $\beta_{1}$. Moreover we can choose $K$ to be amphichiral and $K_{*}$ to be chiral.

The knot $K_{*}$ in this case will be constructed from $K$ by choosing 3 "bands" of a Seifert surface for $K$ and tying them into the shape of a Borromean rings, with a restriction on the 3 bands that they are "essential" (in a sense to be made precise) in the Alexander module. This then becomes very interesting in light of previous work of S. Naik and T. Stanford who showed that any two knots with isomorphic classical Alexander modules and isomorphic classical Blanchfield forms are related by a sequence of such replacements (without the restriction) [NS]; and work of S. Garoufalidis and L. Rozansky, who define a "finite type" isotopy invariant of knots that is affected by precisely this same construction. Their invariant gives information even for Alexander polynomial one knots GR. The work of Naik-Stanford can be interpreted as saying that the construction by which we prove Theorem 10.3 is the only construction necessary (for $n=0$ ) to achieve the full range of values of the triple $\left(\mathcal{A}_{0}(K), \mathcal{B} \ell_{0}(K)\right.$, $\left.\beta_{1}(K)\right)$. 


\section{Infection by a string link}

We discuss an instance of genetic infection of a fixed knot $K$ using an auxiliary string link. This was perhaps first discussed in [CO in the case that the auxiliary link is a boundary link. For our examples it suffices to consider the case where the auxiliary link is the Borromean Rings. This type of Borromean modification has been considered by many other authors including Matveev, Habiro, Goussarov and those mentioned above. Let $\Delta$ be a 2-disk with 3 disjoint open subdisks $\Delta_{1}, \Delta_{2}, \Delta_{3}$, deleted. Consider an embedding of $\Delta$ in $S^{3} \backslash K$ which extends to an embedding $\Delta^{+}$of $D^{2}$ into $S^{3}$. An example is shown in Figure 6. The trivial braid $K \cap\left(\Delta^{+} \times[0,1]\right) \hookrightarrow \Delta^{+} \times[0,1]$ is obtained from the trivial 3 -string braid by forming $\left\{m_{1}, m_{2}, m_{3}\right\}$ parallel strands (and perhaps altering some orientations) where $m_{i}$ is the number of components of $K \cap \Delta_{i}$. From the Borromean rings (written as a 3 -string braid) form the $\left\{m_{1}, m_{2}, m_{3}\right\}$ cable of the Borromean rings and alter orientations consistent with the above. Then replace the trivial braid with this cable of the Borromean Rings. We denote the modified knot by $K_{*}=K(\eta)$ where $\eta$ denotes the triple $\left(\eta_{1}, \eta_{2}, \eta_{3}\right)=\left(\partial \Delta_{1}, \partial \Delta_{2}, \partial \Delta_{3}\right)$ of conjugacy classes of elements of $G=\pi_{1}(E(K))$ as shown in Figure 6 .

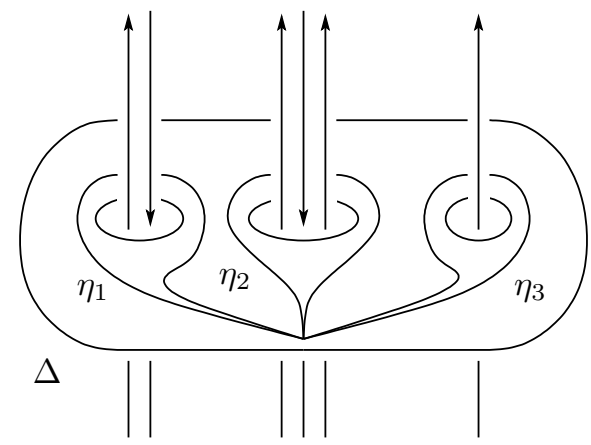

Figure 6: The data required to infect $K$ by a string link along $\left(\eta_{1}, \eta_{2}, \eta_{3}\right)$

Once again this is the same as replacing the solid handlebody $\Delta \times[0,1]$ with the exterior of a 3 -string braid that represents the Borromean Rings.

The Seifert Van-Kampen proof of the following Lemma is left to the reader, it being entirely analogous to that of Theorem 8.1.

Lemma 10.4 If $\eta_{i} \in G^{(n)}, i=1,2,3$ then $\pi_{1}\left(E\left(K_{*}\right)\right) /\left(\pi_{1}\left(E\left(K_{*}\right)\right)\right)^{(n+1)}$ is isomorphic to $\pi_{1}(E(K)) /\left(\pi_{1}(E(K))\right)^{(n+1)}$ preserving peripheral structure. In particular $\mathcal{A}_{i}^{\mathbb{Z}}\left(K_{*}\right) \cong \mathcal{A}_{i}^{\mathbb{Z}}(K)$ for $0 \leq i<n$. 
Now consider the difference $\beta_{n}\left(K_{*}\right)-\beta_{n}(K)$ projected onto $\Omega_{3}\left(P / P^{(n+1)}\right) \cong$ $H_{3}\left(P / P^{(n+1)}\right)$, forgetting the spin structure. We claim that this element is equal to the image of a generator of $H_{3}\left(S^{1} \times S^{1} \times S^{1}\right)$ under the map induced by $\mathbb{Z}^{3} \stackrel{\left(\eta_{1}, \eta_{2}, \eta_{3}\right)}{\longrightarrow} P^{(n)} / P^{(n+1)} \longrightarrow P / P^{(n+1)}$. To establish this, we describe a cobordism, over $P / P^{(n+1)}$, from $\left(M_{K} \amalg S^{1} \times S^{1} \times S^{1}, f_{n} \amalg\left(\eta_{1}, \eta_{2}, \eta_{3}\right)\right)$ to $\left(M_{K_{*}},\left(f_{*}\right)_{n}\right)$. First add a 1-handle to $\partial_{+}$of $\left.\left(M_{K} \amalg\left(S^{1} \times S^{1} \times S^{1}\right)\right) \times[0,1]\right)$. A framed link picture of the new $\partial_{+}$is shown in Figure 7 . Since the meridians of the components of the pictured Borromean rings map to $\left(\eta_{1}, \eta_{2}, \eta_{3}\right)$ in $P / P^{(n+1)}$ we can add three 2-handles $\left\{h_{1}, h_{2}, h_{3}\right\}$ as shown in Figure 8 and still have a cobordism over $P / P^{(n+1)}$. But now the knot in Figure 8 is well known to be equivalent to $K(\eta)$ by first sliding the strands of $K$ which link the attaching circles of $h_{i}$ over the corresponding component of the Borromean Rings until the attaching circles of the $h_{i}$ bound disks intersecting only the Borromean rings and then sliding the strands of $K$ over the $h_{i}$ as needed until completely free.
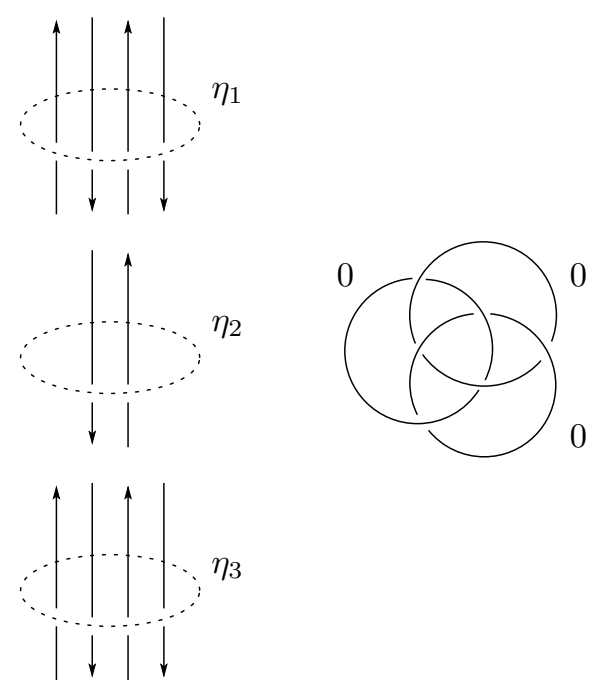

Figure 7

Proof of Theorem 10.3 Let $K$ be a knot with classical Alexander module cyclic of order $p(t) p\left(t^{-1}\right)$ where $p(t)=t^{3}+t-1$. Since $p(t)$ is irreducible and coprime to $p\left(t^{-1}\right)$ there is a unique direct summand $B$ of $\mathcal{A}_{0}(K)$ isomorphic to $\mathbb{Z}\left[t, t^{-1}\right] /\langle p(t)\rangle$. Since $B$ is a free abelian group of rank $3,1 \wedge t \wedge t^{2}$ is a basis of $H_{3}(B)$ and also represents an element $\alpha$ of $H_{3}\left(G / G^{(2)}\right) \cong H_{3}\left(P / P^{(2)}\right)$ under the inclusions. Choose a trivial link $\left\{\eta_{1}, \eta_{2}, \eta_{3}\right\}$ in $S^{3}$, avoiding $K$, representing $\left\{1, t, t^{2}\right\}$ in $G / G^{(2)}$ and perform a Borromean modification to $K$ 


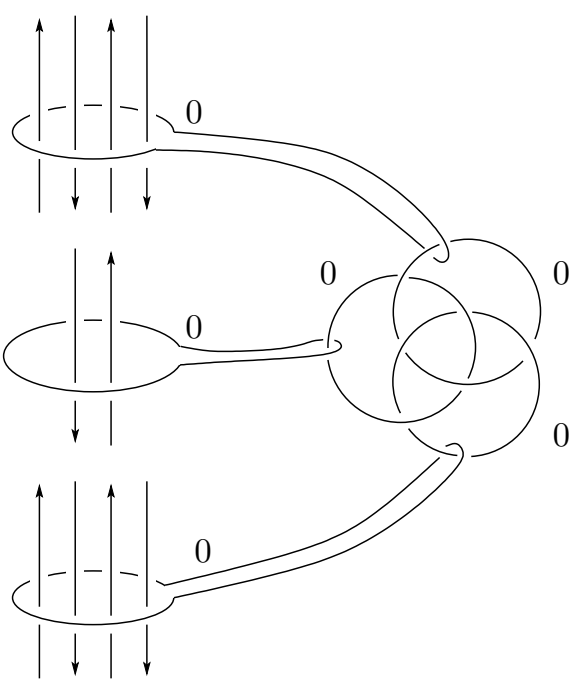

Figure 8

along $\left\{\eta_{1}, \eta_{2}, \eta_{3}\right\}$ as above to arrive at a knot $K_{*}$ that has isomorphic $\mathcal{A}_{0}$. Using the cobordism above we see that $\beta_{1}\left(K_{*}\right)-\beta_{1}(K)=\alpha$. However we must take into account the ambiguity in the definition of $\beta_{1}(K)$. Suppose $f$ is an automorphism of the group $G / G^{(2)}$. Assuming $\beta_{1}\left(K_{*}\right)-f_{*} \beta_{1}(K)=0$ for some $f$, we shall derive a contradiction. Here we are viewing both $\beta_{1}\left(K_{*}\right)$ and $\beta_{1}(K)$ as elements of $H_{3}\left(G / G^{(2)}\right)$. Let $r$ be the canonical retract from $\mathbb{Z}\left[t, t^{-1}\right] /\left\langle p(t) p\left(t^{-1}\right)\right\rangle$ to $B$, inducing a map $r$ from $G / G^{(2)}$ (which is $\mathcal{A}_{0} \rtimes \mathbb{Z}$ to $\pi=B \rtimes \mathbb{Z}$ ). The automorphism $f$ induces an automorphism $g$ of $\pi$ such that $g \circ r=r \circ f$. Combining the two equations above we see that $f_{*} \beta_{1}(K)-\beta_{1}(K)=$ $\alpha$ and hence $r_{*}(\alpha)=r_{*} f_{*} \beta_{1}(K)-r_{*} \beta_{1}(K)=\left(g_{*}-\mathrm{id}\right)\left(r_{*} \beta_{1}(K)\right)$. Consider the Wang sequence

$$
H_{3}(B) \stackrel{t_{*}-\mathrm{id}}{\longrightarrow} H_{3}(B) \stackrel{j_{*}}{\longrightarrow} H_{3}(\pi) \stackrel{\partial}{\longrightarrow} H_{2}(B) \stackrel{t_{*}-\mathrm{id}}{\longrightarrow} H_{2}(B) .
$$

Since $H_{2}(B)$ is free abelian on $\left\{1 \wedge t, 1 \wedge t^{2}, t \wedge t^{2}\right\}$ one can easily calculate that $\left(t_{*}-\mathrm{id}\right)$ is injective on $H_{2}(B)$. Hence $\partial$ is the zero map and $r_{*}\left(\beta_{1}(K)\right)=j_{*}(\beta)$ for some $\beta \in H_{3}(B)$. Recall that, by definition, $r_{*}(\alpha)=j_{*}\left(1 \wedge t \wedge t^{2}\right)$. It follows that $\left(1 \wedge t \wedge t^{2}\right)-\left(g_{*}-\mathrm{id}\right)(\beta)$ lies in the kernel of $j_{*}$ and hence in the image of $\left(t_{*}-\right.$ id). But $H_{3}(B)$ is $\mathbb{Z}$ generated by $1 \wedge t \wedge t^{2}$ so it is easy to calculate that $t_{*}-$ id is zero on $H_{3}(B)$. Moreover since $g_{*}$ is an automorphism of an infinite cyclic group, it equals \pm id. Hence $1 \wedge t \wedge t^{2}=0$ or $1 \wedge t \wedge t^{2}=-2 \beta$, both contradictions. Therefore $\beta_{1}\left(K_{*}\right)$ and $\beta_{1}(K)$ are distinct. Alternatively, we could choose the amphichiral knot $K \#-K$ and form $K_{*}$ by infecting "the 
$K$ part" as above. Then $K_{*}$ is not amphichiral.

\section{Von Neumann higher-order signatures of knots}

One can define higher-order signatures $\rho_{n}, n \geq 0$, for knots using the Von Neumann $\rho$-invariant of J. Cheeger and M. Gromov. In this section these are defined and used to distinguish among knots which have isomorphic localized Alexander modules. These can also be used to detect chirality of knots. Similar signatures were crucial in the work of Cochran-Orr-Teichner [COT1] [COT2] CT].

If $K$ is a knot and $G$ its group, let $M_{K}$ denote the result of zero framed surgery on $K$ and let $P=\pi_{1}\left(M_{K}\right)$. To the $P^{(n+1)}$ covering space of $M_{K}$, Cheeger and Gromov associate a real-valued Von Neumann $\rho$-invariant, which we denote $\rho_{n}(K)$ ChG]. If $-K$ denotes the mirror-image of $K$ then $M_{-K} \cong-M_{K}$ so $\rho_{n}(-K)=-\rho_{n}(K)$ ChG. If $K$ and $K^{\prime}$ are equivalent knots, then $M_{K}$ and $M_{K^{\prime}}$ are (orientation-preserving) homeomorphic so $\rho_{n}(K)=\rho_{n}\left(K^{\prime}\right)$. Hence if $K$ is plus or minus amphichiral then $\rho_{n}(K)=0$ for each $n$. In general it is not known how to compute $\rho_{n}$. However relative signatures $\rho_{n}\left(K_{0}\right)-\rho_{n}\left(K_{1}\right)$ are often easy to compute. Suppose $K_{0}, K_{1}$ are knots such that $P_{0} / P_{0}^{(n+1)} \cong$ $P_{1} / P_{1}^{(n+1)}$ where $P_{i}=\pi_{1}\left(M_{K_{i}}\right)$ as above. Moreover suppose $M_{K_{0}}$ and $M_{K_{1}}$ are bordant over $P / P^{(n+1)}$, as in the previous section, that is there exists a compact oriented 4-manifold $\left(W, \psi: \pi_{1}(W) \longrightarrow P / P^{(n+1)}\right)$ whose boundary is $\left(M_{K_{0}}, \phi_{0}: \pi_{1}\left(M_{K_{0}}\right) \longrightarrow P / P^{(n+1)}\right) \amalg\left(-M_{K_{1}}, \phi_{1}: \pi_{1}\left(M_{K_{1}}\right) \longrightarrow P / P^{(n+1)}\right)$ where $\phi_{i}$ is a composition of the projection $P_{i} \longrightarrow P_{i} / P_{i}^{(n+1)}$ with an arbitrary identification of $P_{i} / P_{i}^{(n+1)}$ with a standard copy called $P / P^{(n+1)}$. Then the relative signature $\rho_{n}\left(K_{0}\right)-\rho_{n}\left(K_{1}\right)$ is equal to the (reduced) $L^{2}$-signature $\sigma_{n}^{(2)}(W)-\sigma(W)$ associated to $\psi$ (see [COT1, Section 5]. This is often calculable. For example, if $n=0$ and $K$ is an Arf invariant zero knot, then $P / P^{(1)} \cong \mathbb{Z}$ and it is known that $\left(M_{K}, \phi_{0}: P \longrightarrow \mathbb{Z}\right)$ is null-bordant, i.e. that $M_{K}$ bounds $(V, \psi)$, and that $\sigma_{0}^{(2)}(K)$ is the integral of the Levine signature function of $K$ and $\sigma(V)$ is the ordinary knot signature COT2. In this sense the $\rho_{n}$ generalize "ordinary" Levine-Tristram signatures associated to the $\mathbb{Z}$-cover of $S^{3} \backslash K$.

The technique of genetic infection may be used to modify a given knot $K$ so subtly that the two have isomorphic $i^{\text {th }}$ localized modules for $i \leq n$ and have isomorphic (integral) modules for $i<n$. The difference in the (integral) modules at the $n^{\text {th }}$ stage can (in many cases) be detected by the $n^{\text {th }}$ relative signature. 
Theorem 11.1 Let $K_{*}=K(\eta, J)$ be the result of genetic infection of $K$ by $J$ along $\eta \in G^{(n)}\left(G=\pi_{1}(E(K))\right)$ as in Section 8, Then

1) $\mathcal{A}_{i}^{\mathbb{Z}}\left(K_{*}\right) \cong \mathcal{A}_{i}^{\mathbb{Z}}(K)$ for $i<n$,

2) If $n>0, \mathcal{A}_{i}\left(K_{*}\right) \cong \mathcal{A}_{i}(K)$ and $\delta_{i}\left(K_{*}\right)=\delta_{i}(K)$ for $i \leq n$,

3) If Arf $J=0$ then $\beta_{i}\left(K_{*}\right)=\beta_{i}(K)$ and $\widetilde{\beta}_{i}\left(K_{*}\right)=\widetilde{\beta}_{i}(K)$ for $i \leq n$,

4) $\rho_{i}\left(K_{*}\right)=\rho_{i}(K)$ for $i<n$,

5) If $\eta \notin P^{(n+1)}$ then $\rho_{n}\left(K_{*}\right)-\rho_{n}(K)$ is the integral of the normalized Levine signature function of $J$. If this real number is non-zero then $K_{*}$ is distinct from $K$ and distinct from the mirror image of $K_{*}$.

Proof Since 1) and 2) were shown in Theorem 8.1 and Theorem 8.2 we begin with 3). Consider the map $f: M_{J} \longrightarrow S^{1}$ induced by the abelianization of $\pi_{1}(E(J))$. Since $\Omega_{3}\left(S^{1}\right) \cong 0$ and $\Omega_{3}^{\text {Spin }}\left(S^{1}\right) \cong \mathbb{Z}_{2}$ as detected by the Arf invariant of $J$, it can be shown that $M_{J}$ is the boundary of a 4 -manifold $V$ with $\pi_{1}(V) \cong \mathbb{Z}$ generated by the meridian of $J$ and such that $V$ extends the usual spin structure on $M_{J}$ if $\operatorname{Arf} J=0$. We may also assume signature $(V)=0$ by connected summing with $\pm \mathbb{C} P(2)$ 's. The boundary of $V$ decomposes into $E(J) \cup\left(S^{1} \times D^{2}\right)$. We form a cobordism $W$ from $M_{K}$ to $M_{K_{*}}$ (or $E(K)$ to $\left.E\left(K_{*}\right)\right)$ as follows. Let $W$ be the 4 -manifold obtained from $M_{K} \times[0,1]$ by identifying $S^{1} \times D^{2} \hookrightarrow \partial V$ with the solid torus neighborhood of $\eta$ in $M_{K} \times\{1\}$ in such a way that $\partial^{+} W=M_{K_{*}}$. Since $\pi_{1}\left(M_{K}\right) \longrightarrow \pi_{1}(W)$ is an isomorphism, $W$ is a cobordism "over" $\pi_{1}\left(M_{K}\right) /\left(\pi_{1}\left(M_{K}\right)\right)^{(i+1)}$ for any $i$. By Theorem 8.1 this quotient is isomorphic to that of $K_{*}$ if $i \leq n$ (since the longitudes are preserved under the map $f$ of Theorem 8.1) and so $\beta_{i}$ and $\widetilde{\beta}_{i}$ agree for $K_{*}$ and $K$ if $i \leq n$. Then $\rho_{n}\left(K_{*}\right)-\rho_{n}(K)$ is equal to the $L^{2}$-signature of $W$ associated the homomorphism $\pi_{1}(W) \cong P \longrightarrow P / P^{(n+1)}$. By additivity of signature [COT1, Lemma 5.9], this is equal to the $L^{2}$ signature of $V$ associated to the map $\pi_{1}(V) \longrightarrow \pi_{1}(W) \longrightarrow P / P^{(n+1)}$. Since $\pi_{1}(V) \cong \mathbb{Z}$ generated by $\eta\left(=\mu_{J}\right)$, if $\eta \notin P^{(n+1)}$, this map is injective. It follows that the $L^{2}$ signature associated to $P / P^{(n+1)}$ is equal to that associated to the map $\pi_{1}(V) \longrightarrow \mathbb{Z}$ (its image) COT1, Proposition 5.13]. But this is the integral of the classical Levine signature function of $J$ over the circle as remarked above [COT2, Appendix]. Note that $\rho_{i}\left(K_{*}\right)=\rho_{i}(K)$ for $i<n$ because in this case the map $\pi_{1}(V) \longrightarrow$ $P / P^{(i+1)}$ is zero and the $L^{2}$-signature of $V$ is equal to its usual signature (which is zero).

Remarks If $n \geq 1$, it is easy to get $\operatorname{genus}\left(K_{*}\right)=\operatorname{genus}(K)$ by choosing $\eta$ in the complement of a minimal genus Seifert surface for $K$. Then $K$ and $K_{*}$ 
also have identical Seifert form. This shows that $\rho_{n}$ is determined neither by the localized modules or $\delta_{i}$ for $i \leq n$, nor by the bordism invariants $\beta_{i}$ for $i \leq n$, nor by the genus. Note that the above proof also establishes part 7) of Theorem 9.4

Question Is $\rho_{n}(K)$ determined by $\mathcal{A}_{n}(K)$ and the $n^{\text {th }}$ linking form $B \ell_{n}$ discussed in the next section?

\section{Higher order Blanchfield linking forms, duality, and the behavior of the longitude}

We will now show that the Blanchfield linking form defined on the classical Alexander module generalizes to linking forms $B \ell_{n}$ on the localized higherorder Alexander modules $\mathcal{A}_{n}$. We see that if $n \neq 1$, we can get a non-singular linking form. If $n=1$ the form is non-singular after killing the longitude. Hence the $\mathcal{A}_{n}$ are self-dual if $n \neq 1$. Recall that $(\mathcal{A}, \lambda)$ is a symmetric linking form if $\mathcal{A}$ is a torsion $\mathcal{R}$-module and

$$
\lambda: \mathcal{A} \longrightarrow \overline{\operatorname{Hom}_{\mathcal{R}}(\mathcal{A}, \mathcal{K} / \mathcal{R})} \equiv \mathcal{A}^{\#}
$$

is an $\mathcal{R}$-module map such that $\lambda(x)(y)=\overline{\lambda(y)(x)}$ (here $\mathcal{K}$ is the field of fractions of $\mathcal{R}$ and Hom, which is naturally a left module, is made into a right $\mathcal{R}$-module using the involution of $\mathcal{R}$ ). The linking form is non-singular if $\lambda$ is an isomorphism.

Theorem 12.1 COT1 Suppose $M$ is a compact, oriented, connected 3manifold with $\beta_{1}(M)=1$ and $\phi: \pi_{1}(M) \longrightarrow \Gamma$ a non-trivial PTFA coefficient system. Suppose $\mathcal{R}$ is a ring such that $\mathbb{Z} \Gamma \subseteq \mathcal{R} \subseteq \mathcal{K}$. Then there is a symmetric linking form

$$
B \ell: H_{1}(M ; \mathcal{R}) \longrightarrow H_{1}(M ; \mathcal{R})^{\#}
$$

defined on the higher-order Alexander module $\mathcal{A}:=H_{1}(M ; \mathcal{R})$.

Proof Note that $\mathcal{A}$ is a torsion $\mathcal{R}$-module by Proposition 3.10 since $\mathcal{K}$ is also the quotient field of the Ore domain $\mathcal{R}$. Define $B \ell$ as the composition of the following maps: the natural map $\pi: H_{1}(M ; \mathcal{R}) \longrightarrow H_{1}(M, \partial M ; \mathcal{R})$, the Poincaré duality isomorphism to $H^{2}(M ; \mathcal{R})$, the inverse of the Bockstein to $H^{1}(M ; \mathcal{K} / \mathcal{R})$, and the usual Kronecker evaluation map to $\mathcal{A}^{\#}$. The Bockstein

$$
B: H^{1}(M ; \mathcal{K} / \mathcal{R}) \longrightarrow H^{2}(M ; \mathcal{R})
$$


associated to the short exact sequence

$$
0 \longrightarrow \mathcal{R} \longrightarrow \mathcal{K} \longrightarrow \mathcal{K} / \mathcal{R} \longrightarrow 0
$$

is an isomorphism since $H^{*}(M ; \mathcal{K}) \cong H_{*}(M, \partial M ; \mathcal{K}) \cong 0$ by Corollary 3.12

We also need to show that $B \ell$ is "conjugate symmetric". The diagram below commutes up to a sign (see, for example, [M] p. 410]), where $B^{\prime}$ is the homology Bockstein

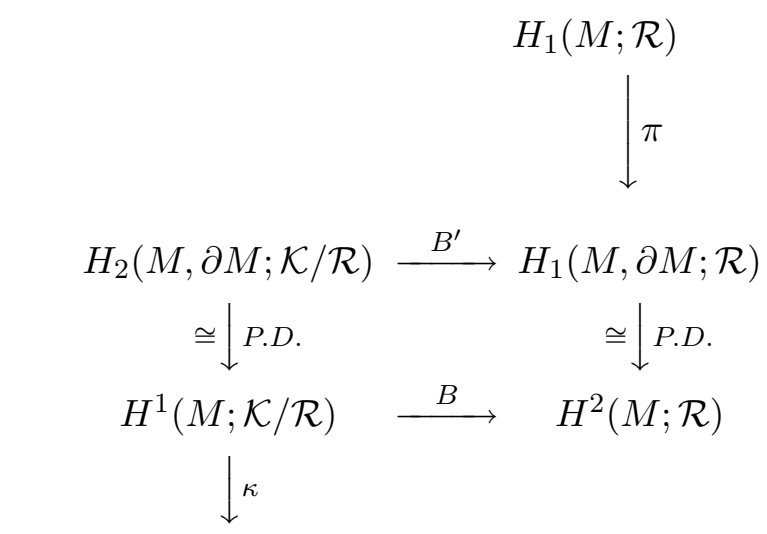

$$
\operatorname{Hom}_{\mathcal{R}}\left(H_{1}(M ; \mathcal{R}), \mathcal{K} / \mathcal{R}\right)
$$

and the two vertical homomorphisms are Poincaré duality. Thus our map $B \ell$ agrees with that obtained by going counter-clockwise around the square and thus agrees with the Blanchfield form defined by J. Duval in a non commutative setting [D, p. 623-624]. The argument given there for symmetry is written in sufficient generality to cover the present situation and the reader is referred to it.

Definition 12.2 The $n^{\text {th }}$-order linking form for the knot $K, B \ell_{n}: \mathcal{A}_{n}(K) \rightarrow$ $\mathcal{A}_{n}(K)^{\#}$, is the linking form above with $R=R_{n}$ (as in Section 4).

Proposition 12.3 The linking form $B \ell: \mathcal{A}_{n}(K) \longrightarrow \mathcal{A}_{n}(K)^{\#}$ is non-singular if $n \neq 1$. If $n=1$ the kernel of $B \ell$ is the submodule generated by the longitude, and there is a non-singular linking form induced on the "reduced" (quotient) module $\mathcal{A}_{1}^{*}(K)$, obtained by killing the longitude.

Corollary 12.4 The localized modules $\mathcal{A}_{n}(K)$ (if $n=1$ use $\mathcal{A}_{1}^{*}(K)$ ) are selfdual. It follows that the higher-order Alexander polynomials $e_{i}^{n}$ and $\Delta_{n}$ of Theorem 5.1] are self-dual (an element $\Delta$ of $R$ is self-dual if it is similar to $\bar{\Delta}$ ). 
Proof of Corollary 12.4 Note that for a finite cyclic module, $A=R / e R$, $\operatorname{Hom}(A, \mathcal{K} / R) \cong R / R e$ and $A^{\#} \cong R / \bar{e} R$. The result then follows from the uniqueness in Theorem 5.1

Proof of Proposition 12.3 The Kronecker map $H^{1}\left(S^{3} \backslash K ; R_{n}\right) \longrightarrow$ $\operatorname{Hom}_{R_{n}}\left(\mathcal{A}_{n}(K), \mathcal{K} / R_{n}\right)$ is an isomorphism since, over the PID $R_{n}$, the usual Universal Coefficient Theorem holds (Remark 3.6.3) and $\operatorname{Ext}_{R_{n}}\left(H_{0}\left(S^{3} \backslash K ; R_{n}\right)\right.$, $\left.\mathcal{K}_{n} / R_{n}\right)=0$ since $\mathcal{K} / R_{n}$ is clearly a divisible $R_{n}$-module and hence an injective $R_{n}$-module by [Ste, I Prop. 6.10]. Thus $B \ell$ is a isomorphism if and only if the $\operatorname{map} \pi: H_{1}\left(S^{3} \backslash K ; R_{n}\right) \longrightarrow H_{1}\left(S^{3} \backslash K, \partial\left(S^{3} \backslash K\right) ; R_{n}\right)$ is an isomorphism. When $n=0$, the map $H_{0}\left(\partial\left(S^{3} \backslash K\right) ; \mathbb{Q}\left[t, t^{-1}\right]\right) \longrightarrow H_{0}\left(S^{3} \backslash K ; \mathbb{Q}\left[t, t^{-1}\right]\right)$ is an isomorphism, implying $\pi$ is onto. Moreover $H_{1}\left(\partial\left(S^{3} \backslash K\right) ; \mathbb{Q}\left[t^{ \pm 1}\right]\right)$ has zero image in $H_{1}\left(S^{3} \backslash K ; \mathbb{Q}\left[t^{ \pm 1}\right]\right)$ since any Seifert surface for $K$ lifts to the $\Gamma_{0}$ ( $\infty$-cyclic) covering space, in other words the longitude $\ell \in G^{(2)}$. Thus $\pi$ is an isomorphism when $n=0$. Now suppose $n \geq 2$. If $K$ has Alexander polynomial 1 then $G^{(1)}$ is a perfect group so $G^{(1)}=G^{(n)}$ for all $n$ and thus $\Gamma_{0}=\Gamma_{n}$ for all $n$ and $\mathcal{A}_{n}=\mathcal{A}_{0}$ for all $n$. The non-singularity then follows from the $n=0$ case. Thus we may assume that $G^{(1)} / G^{(2)} \neq 0$. Below it will be shown that the longitude is non-trivial in $G^{(2)} / G^{(3)}$. In particular the longitude is non-trivial in $\Gamma_{n}=G / G^{(n+1)}$ if $n \geq 2$. Since $\ell \in G^{(1)}$, it follows that $\ell$ is a non-trivial element of $\widetilde{G}_{n+1}$. Therefore $\ell-1$ is a non-zero element of $\mathbb{Z} \widetilde{G}_{n+1}$ and thus is invertible in $R_{n}$. Thus, by (the proof of) Proposition 3.7 $H_{0}\left(\partial\left(S^{3} \backslash K\right) ; R_{n}\right)=R_{n} / R_{n} I=0$, and so $\pi$ is surjective. Moreover since $\ell$ is non-trivial in $\Gamma_{n}$ if $n \geq 2, \pi_{1}\left(\partial\left(S^{3} \backslash K\right)\right)$ embeds in $\Gamma_{n}$ and the induced $\Gamma_{n}$ cover is a union of planes so $H_{1}\left(\partial\left(S^{3} \backslash K\right) ; R_{n}\right)=0$ and $\pi$ is also injective. This finishes the proof of the proposition in the case $n \neq 1$, modulo the proof that $\ell \notin G^{(3)}$ (assuming $G^{(1)} / G^{(2)} \neq 0$ ).

If $n=1$, the situation is more complicated. Let $E=S^{3} \backslash K$ and consider the commutative diagram below where all groups have coefficients in $R_{1}$ unless specified.

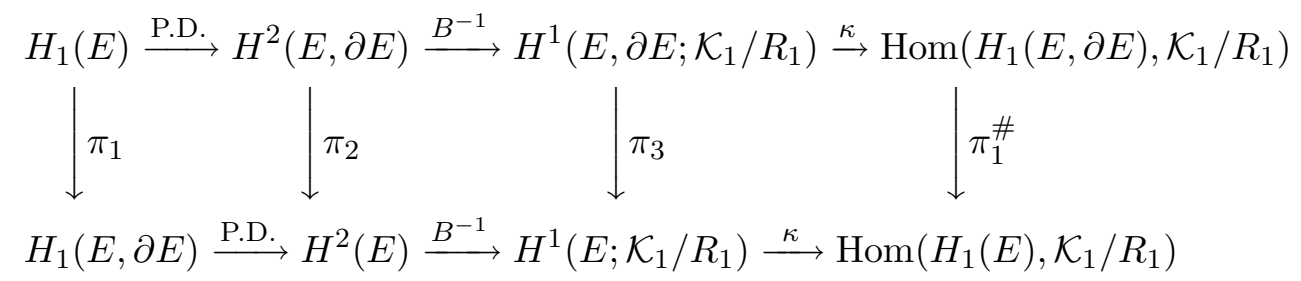

All of the horizontal maps are isomorphisms. Let $f$ (respectively $g$ ) denote the composition of all the maps in the top (bottom) row. Then $B \ell=g \circ \pi_{1}$ and its kernel is precisely the kernel of $\pi_{1}$ which equals the image of $i_{*}: H_{1}(\partial E) \longrightarrow$ 
$H_{1}(E)$. This image is clearly generated by the longitude since the infinite cyclic cover of $\partial E$ is an annulus homotopy equivalent to a circle representing a lift of the longitude. Moreover the induced map $B \ell^{*}$ is thus injective on $\mathcal{A}_{1}^{*} \cong H_{1}(E) / i_{*}\left(H_{1}(\partial E)\right)$. It remains only to show that the image of $g \circ \pi_{1}$ is naturally isomorphic to $\left(\mathcal{A}_{1}^{*}\right)^{\#}$, i.e. $\operatorname{Hom}\left(H_{1}(E) / H_{1}(\partial E), \mathcal{K}_{1} / R_{1}\right)$. The image of $g \circ \pi_{1}$ equals the image of $\pi_{1}^{\#}$ since $f$ is an isomorphism. Consider the commutative diagram below. Since $\bar{\pi}_{1}: H_{1}(E) / H_{1}(\partial E) \longrightarrow H_{1}(E, \partial E)$

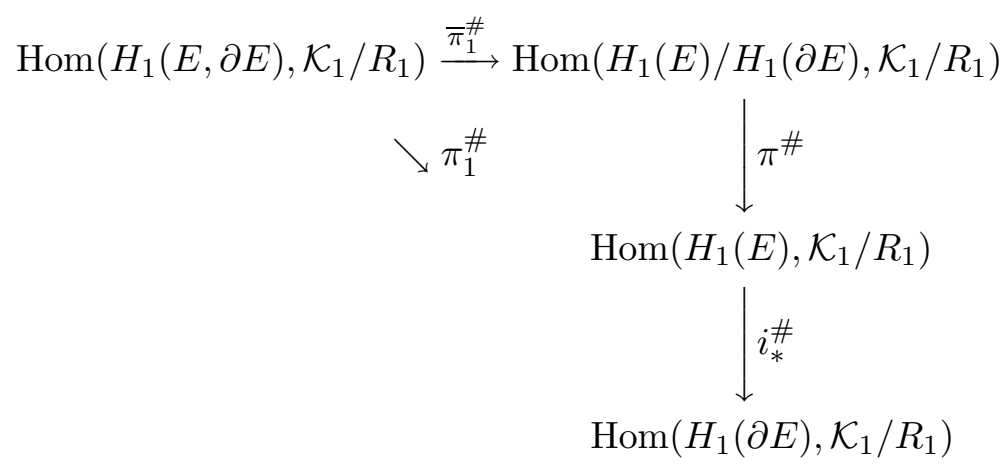

is injective, its dual map $\bar{\pi}_{1}^{\#}$ (the horizontal map above) is surjective since $\operatorname{Ext}_{R_{1}}\left(\ldots, \mathcal{K}_{1} / R_{1}\right)=0$ as remarked earlier in the proof. Therefore the image of $\pi^{\#}$ is contained in the image of $g \circ \pi_{1}$. Note that the image of (the diagonal $\pi_{1}^{\#}$ is contained in the kernel of $i_{*}^{\#}$. But the vertical sequence is exact and $\pi^{\#}$ is injective since Hom is right exact. Thus image $\left(B^{*} \ell\right)=\operatorname{image}\left(\pi_{1}^{\#}\right)=$ $\operatorname{image} \pi^{\#}$, and $\pi^{\#}$ induces an isomorphism between $\left(\mathcal{A}_{1}^{*}\right)^{\#}$ and $\operatorname{image}\left(g \circ \pi_{1}\right)$. Therefore, with this identification, $B \ell$ induces an isomorphism $B \ell^{*}$ between $\mathcal{A}_{1}^{*} \equiv \mathcal{A}_{1} / \operatorname{ker} B \ell$ and $\left(\mathcal{A}_{1}^{*}\right)^{\#}$.

Proposition 12.5 If the (classical) Alexander polynomial of $K$ is not 1, then the longitude of $K$ represents a non-zero class in $G^{(2)} / G^{(3)} \otimes_{\mathbb{Z}\left[G / G^{(2)}\right]} R_{1}$. In particular $\ell \notin G^{(3)}$.

Proof Consider the coefficient system $\phi: G \longrightarrow G / G^{(2)} \equiv \Gamma_{1}$. Let $M$ be the result of zero framed surgery on $K$ so $M=\left(S^{3} \backslash K\right) \cup e^{2} \cup e^{3}$ where the attaching circle of $e^{2}$ is the longitude. Since $\ell \in G^{(2)}$ for any knot, $\phi$ extends to $\pi_{1}(M)$. We may then consider the commutative diagram of exact sequences 
below:

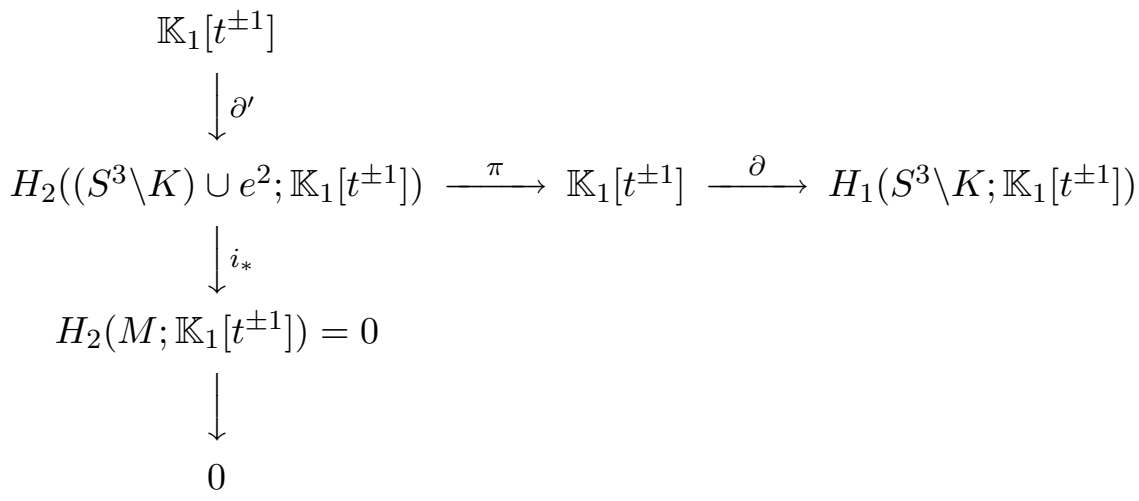

The horizontal sequence is that of the pair $\left(S^{3} \backslash K \cup e^{2}, S^{3} \backslash K\right)$ and the generator of the $\mathbb{K}_{1}\left[t^{ \pm 1}\right]$ may be thought of as $e^{2}$ and its boundary as the class represented by the longitude in $\mathcal{A}_{1}$. Suppose $\ell \in \mathcal{A}_{1} \equiv G^{(2)} / G^{(3)} \otimes_{\mathbb{Z}\left[G / G^{(2)}\right]} R_{1}$ is zero. Then the map $\pi$ would be a surjection. Now consider the vertical exact sequence of the pair $\left(M, S^{3} \backslash K \cup e^{2}\right)$. Here the generator of $\mathbb{K}_{1}\left[t^{ \pm 1}\right]$ may be thought of as the 3 -cell $e^{3}$. We have $H_{2}\left(M ; \mathbb{K}_{1}\left[t^{ \pm 1}\right]\right) \cong H^{1}\left(M ; \mathbb{K}_{1}\left[t^{ \pm 1}\right]\right) \cong$ $\operatorname{Ext}\left(H_{0}\left(M ; \mathbb{K}_{1}\left[t^{ \pm 1}\right]\right), \mathbb{K}_{1}\left[t^{ \pm 1}\right]\right)$. If the Alexander polynomial of $K$ is not 1 then the Alexander module $G^{(1)} / G^{(2)}$ contains some $x \neq e$. Thus $x-1$ lies in the augmentation ideal of $\mathbb{Z} G$ and $\phi(x-1)$ is invertible in $\mathbb{K}_{1}\left[t^{ \pm 1}\right] \equiv R_{1}$ since $x \in \widetilde{G}$ (see Proposition [4.6). Thus $H_{0}\left(M ; \mathbb{K}_{1}\left[t^{ \pm 1}\right]\right.$ ) vanishes by (the proof of) Proposition [3.7 and hence $H_{2}\left(M ; \mathbb{K}_{1}\left[t^{ \pm 1}\right]\right)=0$. Therefore $\partial^{\prime}$ and $\pi \circ \partial^{\prime}$ are epimorphisms. We claim that the diagonal map $\left(\pi \circ \partial^{\prime}\right)$ sends $1 \rightarrow 1-t$. This claim is seen by analyzing how the 3 -cell goes over the 2 -cell twice. This map is clearly not surjective since $1-t$ is not a unit. This contradicts our assumption that the longitude vanished.

\section{Calculation from a presentation of the knot group}

A presentation matrix for $\mathcal{A}_{n}(K)$ can be derived from any finite presentation of $G=\pi_{1}\left(S^{3} \backslash K\right)$.

It is known that, for any regular $\Gamma$ covering space $X_{\Gamma} \rightarrow X$ of a finite complex, the free differential calculus can be used to give a presentation matrix for $H_{1}\left(X_{\Gamma}, \widetilde{x}_{0}\right)$ as a $\mathbf{Z} \Gamma$-module where $\widetilde{x}_{0}$ is the inverse image of a basepoint (see, for example $[\mathrm{H}])$. The torsion submodule of $H_{1}\left(X_{\Gamma}, \widetilde{x}_{0}\right)$ can easily seem to be isomorphic to $H_{1}\left(X_{\Gamma}\right)$. Thus a presentation matrix can be computed for a module whose torsion submodule is $\mathcal{A}_{n}^{\mathbb{Z}}(K)$. The same holds for $\mathcal{A}_{n}(K)$. 
Over a PID, it is theoretically possible to simplify a presentation matrix by appropriate row and column operations until it is diagonal, thus calculating the $\delta_{n}$ (see $\mathrm{Ha}$ ). This necessitates deciding whether or not a given element of the solvable group $G^{(n)} / G^{(n+1)}$ is trivial. Sometimes this is difficult. However note that for $n=1$ this quotient group is merely the classical Alexander module of the knot. Hence there exists a practical algorithm to compute $\mathcal{A}_{1}(K)$. We hope to soon implement this. Details and some sample calculations can be found in Ha.

\section{Questions and open directions}

(1) Find invariants of the higher-order modules which can detect the peripheral structure of a knot.

(2) Find other invariants of the integral modules that are not simply invariants of the localized modules.

(3) Develop effective invariants of the higher-order Alexander polynomials or find ways to reduce their indeterminacy.

(4) Is there a higher-order Seifert form? (The existence of $(t-1)$-torsion has thwarted our efforts on this question.)

(5) Is there a knot $K$ and some $n>0$ for which $\delta_{n}(K)$ is a non-zero even integer? If not then a complete realization theorem for the $\delta_{i}$ can be derived from the techniques of Section 7.

(6) Find higher-order Seiberg-Witten invariants of 3-manifolds that reflect these higher-order modules.

(7) Are the invariants $\delta_{i}$ of finite type?

(8) Prove that for each $n \geq 0$ there exist knots $K$ and $K_{*}$ such that $\mathcal{A}_{i}(K) \cong$ $\mathcal{A}_{i}\left(K_{*}\right)$ for $0 \leq i<n$ but $\beta_{n}\left(K_{*}\right) \neq \beta_{n}(K)$.

(9) The Arf invariant of a knot is determined by its Alexander polynomial which is in turn determined by its Alexander module which is in turn determined by any Seifert matrix. Similarly the Levine-Tristram signatures of a knot are determined by the Alexander module and its Blanchfield form which are in turn determined by a Seifert matrix. Can any such statements be made for the higher-order bordism invariants $\beta_{n}$, modules $\mathcal{A}_{n}^{\mathbb{Z}}$, signatures $\rho_{n}$ and presentation matrices from Section 6 .

(10) Find knots with the same higher-order modules but different linking forms. 
(11) Find ways to compute the $\rho_{n}$.

(12) Apply these ideas to links, string links, braids and mapping class groups.

(13) Do these invariants have any special behavior on other special classes of knots? (for example connected-sums of knots have non-longitudinal $(t-1)$-torsion in $\left.\mathcal{A}_{1}\right)$.

(14) Find applications to contact structures on 3-manifolds (which seem to be closely related to fibering questions).

\section{References}

[A] M.F. Atiyah, Elliptic operators, discrete groups and von Neumann algebras, Asterisque 32-33, 43-72, 1976.

[BW] M. Boileau and S. Wang, Non-zero degree maps and surface bundles over $S^{1}$, J. Diff. Geometry 43, 789-806, 1996.

[Br] K.S. Brown, Cohomology of Groups Springer-Verlag 1982.

[Ch] Jae Choon Cha, Fibred Knots and Twisted Alexander Invariants, preprint 2001, arXiv:math.GT/0109136

[CO] T. Cochran and K. Orr, Homology boundary links and Blanchfield forms: Concordance classification and new tangle-theoretic constructions, Topology 33, no. 3, 397-427, 1994.

[COT1] T. Cochran, K. Orr and P. Teichner, Knot concordance, Whitney towers and $L^{2}$-signatures, Annals of Math.,157,433-519, 2003.

[COT2] T. Cochran, K. Orr and P. Teichner, Structure in the classical knot concordance group, Commentarii Math. Helvetici, 79, 105-123, 2004.

[CT] T. Cochran and P. Teichner, Knot concordance and von Neumann eta invariants, preprint, 2002, Rice University.

[Co1] P.M. Cohn, Skew Fields, Cambridge Univ. Press, Cambridge 1995.

[Co2] P. M. Cohn, Free Rings and their Relations, Second Edition, London Math. Soc. Monographs no. 19, Academic Press, London and New York 1985.

[ChG] J. Cheeger and M. Gromov, Bounds on the von Neumann dimension of $L^{2}-$ cohomology and the Gauss-Bonnet Theorem for open manifolds, J. Diff. Geometry $21,1-34,1985$.

[CTe] J. Conant and P. Teichner, Grope cobordism of classical knots, Topology 43, no. 1, 119-156, 2004.

[Do] Dodziu, Linnell, Matai, Schick, Yates, Approximating L2-invariants, and the Atiyah Conjecture,Dedicated to the memory of Jörgen K. Moser. Comm. Pure Appl. Math. 56, no. 7, 839-873, 2003. 
[D] J. Duval, Forme de Blanchfield et cobordisme d'entrelacs bords, Commentarii Math. Helvetici 61, 617-635, 1986.

[GR] S. Garoufalidis and L. Rozansky, The loop expansion of the Kontsevich integral, abelian invariants of knots and s-equivalence, arXiv:math.GT/0003187. Topology (in press).

[GL] S. Garoufalidis and J. Levine, Homology surgery and invariants of 3-manifolds, Geometry and Topology, vol 5, 551-578, 2001.

[Go] C. Gordon, Some aspects of classical knot theory Knot theory (Proc. Sem., Plans-sur-Bex, 1977), pp. 1-60, Lecture Notes in Math., 685, Springer, Berlin, 1978.

[Hb] K. Habiro, Claspers and finite type invariants of links, Geometry and Topology, vol 4, 1-83, 2001.

[Ha] S. Harvey, Higher Order Polynomial Invariants of 3-manifolds giving lower bounds for the Thurston Norm, preprint, Rice University 2001, arXiv:math.GT/0207014

[H1] J. Harer, How to construct all fibered knots and links, Topology 21 no. 3, 263280, 1982.

[H] J. Hempel, Intersection Calculus on Surfaces with Applications to 3-manifolds, Memoirs of AMS \#282, volume 43, American Mathematical Society, Providence, Rhode Island (1983).

[Hig] G. Higman, The units of group rings, Proc. London Math. Soc. 46, 231-248, 1940.

[Hi2] J. Hillman, Alexander Ideals of Links, LNM \#895, Springer-Verlag, Berlin (1981).

[HK] N. Higson and Kasparov, Preprint 1998.

[HS] P.J. Hilton and U. Stammbach, A Course in Homological Algebra SpringerVerlag, 1971, New York.

[HoS] J. Howie and H.R. Scheebeli, Homological and topological properties of locally indicable groups, Manuscripta Math. 44, no. 1-3, 71-93, 1983.

[J] N. Jacobson, The Theory of Rings, American Math. Soc., New York, 1943.

[Ka] Akio Kawauchi, Almost identical imitations of $(3,1)$-dimensional manifold pairs, Osaka J. Math. 26, 743-758, 1989.

[Ki] R. Kirby, The calculus of framed links in $S^{3}$, Inventiones Math. 145, 35-56, 1978.

[KL] P. Kirk and C. Livingston, Twisted Alexander invariants, Reidemeister torsion and Casson-Gordon invariants, Topology 38, no. 3, 635-661, 1999.

[Kr] P. Kronheimer, Embedded Surfaces and Gauge Theory in Three and Four Dimensions, Surveys in differential geometry, Vol. III, Int. Press, 243-298, 1998. 
[Kr2] P. Kronheimer, Minimal genus in $S^{1} \times M^{3}$, Inventiones Math. 134, 363-400, 1998.

[L1] J. P. Levine, Knot cobordism groups in codimension two, Comm. Math. Helv. 44, 229-244, 1969.

[L3] J. P. Levine, Knot Modules, I, Trans. Amer. Math. Soc. 229, 1977, 1-50.

[Lew] J. Lewin, A note on zero divisors in group rings, Proc. Amer. Math. Soc. 31, 1972, 357-359.

[Ma] V. Mathai, Spectral flow, eta invariants and von Neumann algebras, J. Functional Analysis 109, 442-456, 1992.

[M] J. Munkres, Elements of Algebraic Topology, Addison-Wesley, Menlo Park CA, 1984.

[M2] J. Munkres, Topology, 2nd edition, Prentice Hall, Saddle River, N.J. (2000).

[P] D. Passman, The Algebraic Structure of Group Rings, John Wiley and Sons, 1977, New York.

[NS] S. Naik and T. Stanford, A move on diagrams that generates $S$-equivalence of knots, arXiv:math.GT/9911005, to appear in J. Knot Theory and its Applications.

[Ra2] A. Ranicki, Exact Sequences in the Algebraic Theory of Surgery, Princeton University Press, 1981, Princeton New Jersey.

[Ru] S. Roushon, Topology of 3-manifolds and a class of groups, preprint 2002, arXiv:math.GT/0209121

[Sta] J. Stallings, Constructions of fibered knots and links, AMS Proceedings of Symposia in Pure Math. 32, 1978, 55-60.

[St] J. Stallings, Homology and Central series of Groups,

[Ste] B. Stenström, Rings of Quotients, Springer-Verlag, 1975, New York.

[Sti] A. Stoimenow, Vassiliev invariants on fibered and mutually obverse knots, J. Knot Thry. Ram. 8 (1999), no. 4, 511-519.

[Str] R. Strebel, Homological methods applied to the derived series of groups, Comment. Math. 49, 1974, 302-332.

[W] G.W. Whitehead, Elements of Homotopy Theory, Graduate Texts in Mathematics \#61, Springer-Verlag, 1978, New York.

[Wa] C. T. C. Wall, Surgery on Compact Manifolds, London Math. Soc. Monographs 1, Academic Press 1970.

Department of Mathematics, Rice University

6100 Main Street, Houston, Texas 77005-1892, USA

Email: cochran@rice.edu

Received: 17 March 2004 